\title{
PLATFORM COMPETITION, COMPATIBILITY, AND SOCIAL EFFICIENCY
}

\author{
Ramón Casadesus-Masanell \\ Francisco Ruiz-Aliseda
}


The Public-Private Center is a Research Center based at IESE Business School. Its mission is to develop research that analyses the relationships between the private and public sectors primarily in the following areas: regulation and competition, innovation, regional economy and industrial politics and health economics.

Research results are disseminated through publications, conferences and colloquia. These activities are aimed to foster cooperation between the private sector and public administrations, as well as the exchange of ideas and initiatives.

The sponsors of the SP-SP Center are the following:

- Accenture

- Ajuntament de Barcelona

- Caixa Manresa

- Cambra Oficial de Comerç, Indústria i Navegació de Barcelona

- Consell de l'Audiovisual de Catalunya

- Departamento de Economía y Finanzas de la Generalitat de Catalunya

- Departamento de Innovación, Universidades y Empresa de la Generalitat de Catalunya

- Diputació de Barcelona

- Endesa

- Fundació AGBAR

- Garrigues

- Mediapro

- Microsoft

- Sanofi Aventis

- VidaCaixa

The contents of this publication reflect the conclusions and findings of the individual authors, and not the opinions of the Center's sponsors. 


\title{
PLATFORM COMPETITION, COMPATIBILITY, AND SOCIAL EFFICIENCY.
}

\author{
Ramón Casadesus-Masanell ${ }^{1}$ \\ Francisco Ruiz-Aliseda ${ }^{2}$
}

\begin{abstract}
Katz and Shapiro (1985) study systems compatibility in settings with one-sided platforms and direct network effects. We consider systems compatibility in settings with two-sided platforms and indirect network effects to develop an explanation why markets with two-sided platforms are often characterized by incompatibility with one dominant player who may subsidize access to one side of the market. We find that incompatibility gives rise to asymmetric equilibria with a dominant platform that earns more than under compatibility. We also find that incompatibility generates larger total welfare than compatibility when horizontal differences between platforms are small.
\end{abstract}

Keywords: network. industries, platforms, markets.

\footnotetext{
* We are very grateful to Xiang Ao, Bruno Cassiman, Jim Dana, Jan Eeckhout, Juanjo Ganuza, Andrei Hagiu, Hanna Ha laburda, Andres Hervas-Drane, Ig Horstmann, Sjaak Hurkens, Doh-Shin Jeon, Gaston Llanes, Matthew Mitchell, Karl Schlag, Maxim Sinitsyn, Eric Van den Steen, Timothy Van Zandt, Birger Wernerfelt, Glen Weyl and seminar participants at Universitat Aut_onoma de Barcelona, Imperial College's conference on \Platforms, Markets and Innovation," UPF, HBS, IESE, MIT, Rotman, and the 2009 IIOC at Northeastern for helpful comments. Part of this research was conducted while the second author was visiting INSEAD, and he would like to thank all the members of this institution for their hospitality during his stay, as well as for _nancial support for his research. CasadesusMasanell is grateful to the HBS Division of Research. We gratefully acknowledge _nancial support from the NET Institute (www.netinst.org) and the Kau_man Foundation and IESE Business School's Public-Private Sector Research Center.
}

\footnotetext{
${ }^{1}$ Associate Professor of Harvard Business School.

${ }^{2}$ Assistant Professor of Universitat Pompeu Fabra.
} 


\section{Introduction}

The last three decades have witnessed unprecedented growth in network industries such as video games, computers, credit cards, media, or telecommunications. These industries are often organized around physical or virtual platforms that enable distinct groups of agents to interact with one another and are commonly referred to as two-sided markets or markets with two-sided platforms (Evans, 2003, and Evans and Schmalensee, 2007). An operating systems developer such as Microsoft, for example, provides a software platform that makes possible the completion of value-creating transactions between independent software vendors and users.

It is now well-known that platforms are characterized by the presence of inter-group network effects and that these constitute a central feature affecting pricing by platform providers. For example, when pricing its operating systems and software development kits, Microsoft must take into account that the larger the number of applications expected to run on Windows, the more willing users are to adopt it. Likewise, developers' incentives to write Windows applications grow with the number of users who are expected to adopt that operating system.

A key attribute of these markets that determines the intensity and scope of network effects is whether competing platforms are compatible or not. In a seminal paper, Katz and Shapiro (1985) study systems compatibility in markets with one-sided platforms.1 The literature on markets with two-sided platforms, however, has largely ignored the effects of platform (in)compatibility on market outcomes, which constitutes the focus of our paper.

The contribution of this paper is to develop an explanation of why markets with twosided platforms are often characterized by incompatibility with one dominant player who may choose to subsidize access to one side of the market. Prominent examples include the personal computer or digital music distribution industries, which are dominated by Microsoft's Windows and Apple's iTunes, respectively. At the normative level, we shed light on the nature of the social inefficiencies that competitive pricing in markets with two-sided platforms generate.

We extend the Katz-Shapiro framework to model a situation in which two platform providers mediate between developers of products based on the platform and users of such products. This may be representative of hardware/software industries such as personal computers, smart phones, or videogames. Developers and users first trade with the platform

\footnotetext{
${ }^{1}$ In their setting, platforms set access prices to users but not to software developers. Moreover, there is no transaction between independent software vendors and users.
} 
providers by adopting one of the two platforms. Platforms are assumed to be horizontally differentiated from the users' viewpoints. After having gained access to one platform, users buy applications from developers under oligopolistic conditions that, to a large extent, depend on the pricing structures set by the platform providers.

We compare the nature of platform competition under application compatibility and incompatibility. Under compatibility, active developers can sell their applications to all the users, regardless of the platform they have adopted. ${ }^{2}$ As a result, developers do not benefit from platform-specific network effects and perceive both platforms as homogeneous in that they give access to the same pool of users and number of competitors. Platform homogeneity leads to intense competition for developers: platforms set developer access prices equal to the marginal cost to serve them. Marginal cost pricing implies plentiful entry by developers and low application prices. Under compatibility, platforms cannot vertically differentiate based on the number of applications because users foresee having access to a unique pool of applications. However, because platforms are assumed to be horizontally differentiated, platform providers have some pricing power on the user side. Moreover, because application prices are low in this case, there is large potential value that can be extracted from users through platform access prices. Put differently, access prices to users end up being high because (i) platforms are differentiated horizontally and (ii) inter-group network effects are exploited very intensely due to fierce competition for developers. We also find that compatibility gives rise to a unique equilibrium and that this equilibrium is symmetric.

The nature of platform competition changes dramatically when platforms are incompatible. To begin, there is a unique symmetric equilibrium under incompatibility, but there are also asymmetric equilibria. The symmetric equilibrium under incompatibility exhibits softer price competition for developers compared to the case in which platforms are compatible. Less intense competition for developers leads to reduced entry and higher application prices. This implies that less user surplus is generated and, thus, access prices to users end up being lower than in the case of compatible platforms $3^{3}$ In fact, when horizontal differ-

\footnotetext{
${ }^{2}$ More precisely, under (full) platform compatibility, an application sold by a developer is functional on any platform, no matter with which platform provider the developer traded in the first period. Under platform incompatibility, an application sold by a developer is valuable only on the platform sold by the platform provider with which such developer traded.

${ }^{3}$ That platform compatibility softens price competition for users in a setting with two-sided platforms is consistent with earlier results. Katz and Shapiro (1985), for instance, derive this result in a setting with network effects and one-sided platforms and Matutes and Regibeau (1988) and Economides (1989) in the absence of network effects.
} 
entiation between platforms is mild and competition for users intense, platform providers end up subsidizing user entry. An important feature of the symmetric equilibrium under incompatibility is that it results in lower platform profits than under compatibility, even if platform-access fees charged to developers are above marginal cost.

Incompatibility is a pervasive feature of many markets with two-sided platforms. This raises the question of why platform providers do not make their platforms compatible if equilibrium profits are higher in that regime. To address this question we study some asymmetric equilibria that are present under incompatibility. In these equilibria, users and developers form expectations favorable to one of the platforms, and hence such platform is able to corner the market by pricing the competitor out. When horizontal differentiation is weak, such equilibria yield higher profits for the winning platform compared to profits in the unique equilibrium under compatibility. Hence, we find that it is the quest for market dominance that prevents providers of platforms from agreeing to make them compatible. We conclude that incompatibility is associated with market dominance, as happens in Katz and Shapiro (1985). Unlike their one-sided setting, however, our model suggests that tipping may be grounded upon user subsidization. We also show that profits for the dominant platform are decreasing in the strength of horizontal differentiation. In fact, when horizontal differentiation is strong, profits in the symmetric incompatible equilibrium may be larger than those of the dominant platform in the asymmetric case. The reason is that stronger differentiation makes it harder for a platform to corner the market, since it has to attract users whose appeal for such a platform is lower.

Our model also allows for detailed social welfare analysis. We find that when platforms are incompatible, the underexploitation of the inter-group network effects originated on the user side results in fewer developers than socially desirable. Because the providers of incompatible platforms cannot capture all the gains that accrue to users if more developers enter, there is no point in promoting too much entry by developers. The result of insufficient entry by developers is reversed under platform compatibility. The fierce price competition for developers that takes place when platforms are compatible (marginal cost pricing) together with the presence of fixed entry costs for developers, results in too many developers relative to what is socially desirable.

As in Katz and Shapiro (1985), we find that a welfare-maximizing social planner prefers compatibility over incompatibility if she can control access prices because welfare-enhancing network effects can be generated at a lower cost under compatibility. However, the social welfare that arises when platforms compete against one another (in the absence of a social 
planner) may be larger with incompatibility. We find that the social welfare comparison is ambiguous when platforms play the unique symmetric equilibrium under compatibility and incompatibility. Compatibility leads to more intense exploitation of inter-group network effects but also to excessive entry. The trade off between the benefits of network effects and the costs of entry is resolved differently depending on the intensity of users' preferences for the horizontal attributes of platforms. We also find that competition under compatibility may lead to lower welfare than market dominance by an incompatible platform. Specifically, a monopolistic platform subject to a credible threat of entry is socially desirable if horizontal differentiation is not too strong.

\section{$1.1 \quad$ Literature}

We contribute to the literature on systems compatibility and oligopolistic competition, initiated by Katz and Shapiro's (1985) path-breaking work and continued by, amongst others, Katz and Shapiro (1986), Economides and Flyer (1997), Crémer, Rey and Tirole (2000), and Malueg and Schwartz (2006). Katz and Shapiro (1994) present a detailed literature review.

Our paper is most closely related to Katz and Shapiro (1985) but differs in several important respects. Our setup deals with two-sided platforms that are horizontally differentiated and that play an access pricing game, while theirs studies one-sided platforms that are undifferentiated and that play a quantity game. Their setting is most appropriate for the study of compatibility and incompatibility in environments with direct network effects, while ours intends to capture situations where indirect network effects are at play. Because we consider two-sided platforms, we are able to draw additional conclusions on the nature of platform competition and its impact on welfare under the different intellectual property regimes that we consider. These additional results are not straightforward extensions of Katz and Shapiro's (1985) one-sided setting. For example, we find that platform providers compete fiercely for developers when platforms are compatible and for users when they are incompatible. In fact, when horizontal differences across platforms are feeble, incompatible platforms are sold below marginal cost to users. Our model predicts that user subsidization may occur only when platforms are incompatible; compatible platforms are never sold below cost to either side. These results, which appear consistent with anecdotal evidence that platforms such as videogame systems are typically priced below marginal cost for users, can only be obtained in a model that explicitly considers both sides of the market. Another aspect that our two-sided setting allows us to study are the effects of developer entry on social efficiency, which differ depending on whether platforms are compatible or not. 
Our modeling approach is that of the recent literature on intermediation by firms in two-sided markets. Pioneering work by Spulber (1996, 1999) examines how firms establish markets acting as intermediaries between buyers and sellers. The platforms that we study also establish markets as they bring users and developers together. This literature has flourished partly on the basis of industry-specific models. Rochet and Tirole (2003), for example, is inspired by the credit card market, Armstrong (2006) captures well the economics of shopping malls or newspapers, and Hagiu (2009) maps to competition between providers of videogame systems. General results have been derived by Spulber (2006), however, who models centralized and decentralized two-sided markets through network theory. Likewise, Rochet and Tirole (2006) propose a formal definition of two-sided markets and present a general framework for the analysis of such markets. Finally, Weyl (2009) develops a general theory of monopoly pricing of networks with weak constraints on the nature of user heterogeneity.

Within this literature, our paper is closest to those in which agents on both sides first trade with the platform providers and then with each other. Caillaud and Jullien (2001, 2003) examine matchmaking intermediation services, such as those provided by dating or real estate agencies, in a model with ex ante identical matchmakers that bear no fixed costs. These papers dealing with incompatible platforms assume that once a match is made, agents realize all gains from trade, and pay special attention to equilibria in which a platform provider prices a competitor out of the market. Such dominant firm equilibria are supported by optimistic rational expectations of agents on both sides of the market, according to which every agent expects everyone else to interact with the dominant firm. Our paper also highlights the importance of agents' expectations in the emergence of dominant firm equilibria in a somewhat related context. In our model, however, trade between users and developers is not efficient and several features of the downstream market structure are endogenously determined.

Our paper is also related to Hagiu (2009), which pioneers the analysis of how platform pricing structures are affected by consumers' preferences for product variety in a setting with monopolistically competitive developers. In a related paper, Hagiu (2006b) focuses on the efficiency of open (or free access) vs. proprietary platforms, a topic which is of independent interest to that of platform (in)compatibility. Indeed, platform compatibility is studied by a few recent papers dealing with two-sided platforms. Orman (2008) studies the effects of compatibility on competition between proprietary and open two-sided platforms and shows that compatibility may increase profits for the proprietary platform. We focus on the case 
where both competing platforms are proprietary. Miao (2007) studies two-sided platforms composed of two components supplied by different producers (such as TV sets and TV broadcasting equipment) and examines suppliers' incentives to provide compatibility within platforms. In contrast, our analysis deals with integrated platform suppliers and is concerned with application compatibility between platforms.

Our paper takes up one of the extensions suggested by Carrillo and Tan (2006). Using their terminology, we analyze the equilibrium outcome of a game in which competing platforms can choose between complete compatibility without standardization and incompatibility. If the equilibrium under incompatibility is characterized by both platforms being active, we show that platforms would have a strong incentive to negotiate complete compatibility without standardization $4^{4}$ However, if incompatibility results in a single platform dominating the market in equilibrium (an outcome that is more likely when platform horizontal differentiation is low), then there is no incentive to negotiate complete compatibility without standardization or complete standardization.

The paper is organized as follows. Section 2 describes the two-period platform competition model. In sections 3 and 4 we study competitive interaction between compatible and incompatible platforms, respectively, focusing on symmetric equilibria. Section 5 studies asymmetric equilibria and compares profits and welfare under both intellectual property regimes. Concluding remarks are offered in Section 6.

\section{The model}

Consider a platform provider, labeled by $i$, that mediates between developers of products based on its platform and users of such products. The platform provider could be a supplier of operating systems, in which case developers would be independent software vendors and users would be individuals or firms that make use of such applications. The platform provider sets access prices for both, users and developers. In the case of operating systems providers, the price charged to users is that of the operating system, while that for developers is the price of the software development kit or a license fee.$^{5}$ We denote the access price paid by users by $p_{i}^{U}$ and that paid by developers by $p_{i}^{D}$. After users and developers have transacted with the platform provider, developers compete against one another to sell their applications.

\footnotetext{
${ }^{4}$ The incentive to achieve complete standardization (i.e., having compatible platforms which are less differentiated) would be lower because of platform homogenization.

${ }^{5}$ Introducing royalties per unit of application sold by developers makes the analysis intractable. Notwithstanding, we discuss the likely role of royalties in the conclusion.
} 
A forward-looking platform provider sets $p_{i}^{U}$ and $p_{i}^{D}$ to maximize profits taking into account the resulting structure of the market for applications.

To analyze this situation formally when two platform providers exist, we set up the following two-period game which extends Church and Gandal's (1993) approach to a market with two-sided platforms. The first period consists of two stages. In the first stage, platform provider $i \in\{1,2\}$ posts access prices $\left(p_{i}^{U}, p_{i}^{D}\right)$. In the second stage, all potential users and developers simultaneously decide whether or not to trade with one of the platform providers. In the second period, developers who traded with platform provider $i$ sell applications to users who own platform $i$ (in the case of platform incompatibility) or any of the two platforms (in the case of compatibility).

As usual in multi-period contexts, we focus on (pure strategy) subgame-perfect equilibria assuming that all agents have rational expectations. We thus solve the model by backwards induction. Figure 1 illustrates the timing of the game for the incompatible case. The timing for the compatible case is similar.

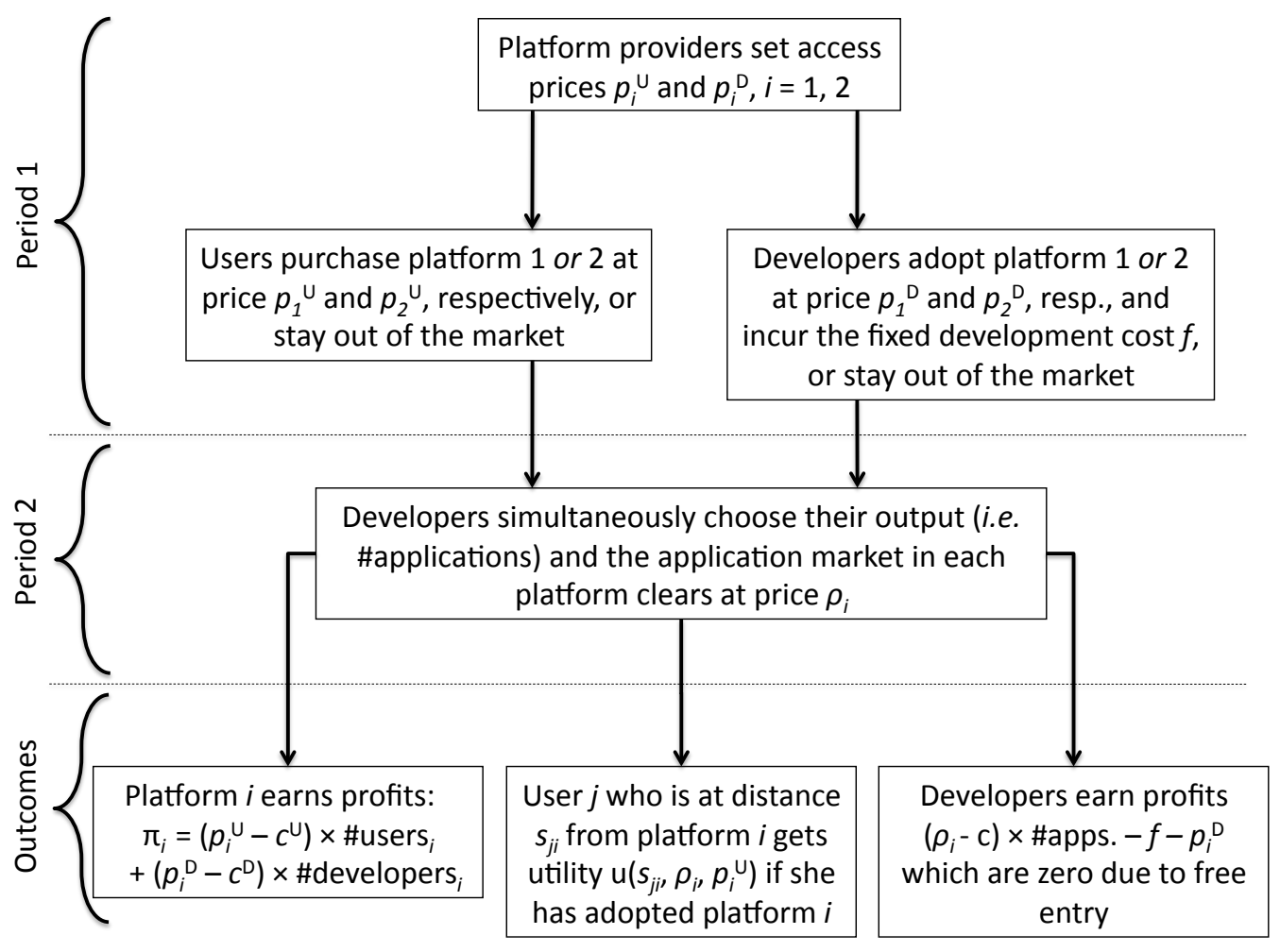

Figure 1: Timing

We make the following assumptions about each player group:

Platform providers. There are two competing platforms that have no stand-alone 
value (e.g., an operating system is valuable only if there are applications that can run on it). The (constant) marginal cost $c^{U}$ of producing copies of a platform is normalized to zero, so $p_{i}^{U}$ is to be interpreted as a mark-up. We allow for the possibility that there may be a positive cost of serving developers (these costs are related to the provision of development kits and/or the structuring of licensing contracts). We assume that these costs are identical across platform providers, and we denote them by $c^{D} \geq 0$.

Users. Users are assumed to have unit demands for platforms and linear demands for applications, which are assumed to be homogeneous goods to simplify the analysis by sidestepping issues related to product variety ${ }^{6}$ Specifically, we assume that a unit mass of (potential) users is uniformly distributed along a Hotelling segment of unit length. We suppose that the platform provider indexed by 1 is located at the left end of the segment, whereas that indexed by 2 is located at the right end. Platform providers differ in their location on the segment but are otherwise identical. Inspired by Novshek (1980), we assume that user $j$ located at distance $s_{j i} \in[0,1]$ from platform $i$ has the following demand for applications sold at price $\rho_{i}$ if she has acquired platform $i: q_{j}\left(\rho_{i}, s_{j i}\right)=\left(1-t s_{j i}\right)\left(a-b \rho_{i}\right)$ (where both $a$ and $b$ are positive constants) 7 In combination with the heterogeneous locations of users on $[0,1]$, parameter $t>0$ captures the intensity of horizontal differences regarding the perceived performance of the platform by the time it is used with the applications sold by developers: every user has a different demand function for applications, and hence users are heterogeneous even after acquiring a platform 8 We let $x_{i}$ denote the measure of users who trade with platform provider $i$ and assume that $t<2 / 3$ to guarantee that the market is covered 9 Therefore, we shall focus on those situations in which $x_{1}=1-x_{2}$.

It is worth noting that departing from the widely used model of unit demand for ap-

\footnotetext{
${ }^{6}$ The main arguments are not dependent on the assumption of homogeneity. We discuss the implications of product variety for pricing structures in the concluding section.

${ }^{7} \mathrm{~A}$ relevant property of this specification is that the sensitivity of user $j$ 's demand to changes in the price of applications does not depend on differences in the perceived performance of the two competing platforms (i.e., it does not depend on $s_{j i}$ ). This property is useful in that it simplifies how first-period behavior affects competitive behavior in the second period (e.g., the equilibrium prices of applications do not depend on the number of users attracted by a platform). More complete models must await further research on these issues which seem far from being easily tractable.

${ }^{8}$ In our setting, horizontal differences across platforms persist even if they are compatible. For instance, a videogame console may load games faster and provide higher definition video and sound compared to another one but may consume more power in doing so. If users differ in the relative weight that they assign to loading speed, video and sound definition, and power consumption, then the platforms will be horizontally differentiated even if they can both run the same applications. We discuss in Subsection 5.2.3 how our results would be affected when horizontal differentiation is weaker under compatibility.

${ }^{9}$ This is standard in Hotelling models of horizontal differentiation. The analysis when the market is not covered is available from the authors.
} 
plications allows us to perform social welfare analysis even if all users purchase one of the platforms and the market is completely covered. In addition, in markets such as video games, personal computers, or smart phones, users often purchase one platform only but buy many applications. Therefore, this aspect of our model is closer to the phenomenon than a specification where users have unitary demands for both, platforms and applications.

Developers. We suppose that there exist infinitely many potential developers of applications, all ex ante identical, and free entry. Those who have traded with one of the platform providers in the first period must also incur a sunk setup cost $f \geq 0$ to become active in the second period. Developers active in the second period produce applications at non-negative constant marginal cost $c$, assumed to be smaller than $a / b$ to avoid making the analysis trivial, and compete ̀̀ la Cournot. Cournot competition can be interpreted as a reduced-form for simultaneous capacity choice by developers followed by simultaneous capacity-constrained price competition amongst them, as shown by Kreps and Scheinkman (1983). We use $n_{i}$ to denote the number of developers that trade with platform provider $i$. As customary in the oligopolistic entry literature (Suzumura and Kiyono, 1987), we will usually ignore the integer problem and treat the number of developers as a continuous variable.

Cournot competition captures the fact that developers best-respond to one another when making profit-maximizing choices. Given the concentration levels in developer communities such as those in video games or personal computers, ${ }^{10}$ it is likely that players do take into account each others' actions when choosing their strategies. Our model reflects this. In addition, the original work by Katz and Shapiro (1985) on which we build assumes that platforms play an output game with homogeneous platforms. We make a first step towards greater realism by adding horizontally differentiated platforms that deal with two sides. In the conclusion (Section 6) we discuss the effect of adding heterogeneous applications to our framework 11

In what follows, we distinguish two situations, depending on whether platforms are compatible or not. Platforms are compatible if any given application can be used in either platform. Platforms are incompatible if any given application can only be used in the platform for which it was originally developed.

\footnotetext{
${ }^{10}$ For instance, the top seven game developers (in terms of market share) accounted for almost two thirds of industry sales in the US in 2004.

${ }^{11}$ Models with both, heterogeneous applications and strategic behavior by developers, have proven intractable.
} 


\section{Compatible platforms}

As mentioned above, the game is solved by backwards induction. We begin by solving for the second period outcomes, given $\left\{\left(p_{i}^{U}, p_{i}^{D} ; x_{i}, n_{i}\right)\right\}_{i=1}^{2}$. Under compatibility any one of the $n_{i}$ developers who have traded with platform provider $i \in\{1,2\}$ sells applications that can be used by users of the competing platform. User demand for the homogeneous good sold by developers depends on the location of the user and the platform that she uses. Letting $x_{1}$ denote the location of the marginal user who purchased platform provider 1's platform, it is immediate that those users to the left of $x_{1}$ must have traded with 1 as well by monotonicity. Similarly, letting $x_{2}$ denote the distance between platform provider 2 and the marginal user who purchased provider 2's platform, the users in between must also have traded with 2. In addition, note that developers produce homogeneous goods regardless of the platform provider they traded with in the first period, so they must charge the same market price $\rho$ as dictated by the properties of standard Cournot competition. Because the demand for applications of user $j$ located at distance $s_{j i} \in\left[0, x_{i}\right]$ from platform $i$, is given by $q_{j}\left(\rho, s_{j i}\right)=\left(1-t s_{j i}\right)(a-b \rho)$, it follows that aggregate demand for applications

equals $Q(\rho)=\int_{0}^{x_{1}} q_{j}\left(\rho, s_{j 1}\right) d j+\int_{0}^{x_{2}} q_{j}\left(\rho, s_{j 2}\right) d j$, and hence aggregate demand for compatible applications given a market price $\rho$ is equal to

$$
Q(\rho)=\left[\left(x_{1}-\frac{t x_{1}^{2}}{2}\right)+\left(x_{2}-\frac{t x_{2}^{2}}{2}\right)\right](a-b \rho)
$$

and inverse demand is as follows:

$$
\rho(Q)=\frac{a}{b}-\frac{2 Q}{b\left[\left(2 x_{1}-t x_{1}^{2}\right)+\left(2 x_{2}-t x_{2}^{2}\right)\right]} .
$$

Therefore, second-period Cournot competition with $n_{1}+n_{2} \equiv N$ developers yields the following equilibrium price for an application:

$$
\rho^{c o m}=\frac{a+b c N}{b(N+1)}
$$

Every developer sells $q^{\text {com }}$ applications, where

$$
q^{c o m}=\frac{\left[\left(2 x_{1}-t x_{1}^{2}\right)+\left(2 x_{2}-t x_{2}^{2}\right)\right](a-b c)}{2(N+1)},
$$


and makes profits equal to

$$
\pi^{c o m}=\frac{\left[\left(2 x_{1}-t x_{1}^{2}\right)+\left(2 x_{2}-t x_{2}^{2}\right)\right]}{2 b}\left(\frac{a-b c}{N+1}\right)^{2} .
$$

For fixed $\left\{\left(p_{i}^{U}, p_{i}^{D}\right)\right\}_{i=1}^{2}$, the effect of compatibility on developers is two-fold. On the one hand, developers have access to a larger pool of users than if platforms were not compatible. On the other, they face more intense competition.

If user $j$ has traded with platform $i$ and is located at distance $s_{i j}$ from such platform, then she attains the following second-period utility:

$$
u_{j}^{c o m}\left(s_{j i}\right)=\int_{\rho^{c o m}}^{a / b}\left(1-t s_{j i}\right)(a-b \rho) d \rho=\frac{\left(1-t s_{j i}\right)\left(a-b \rho^{c o m}\right)^{2}}{2 b}=\frac{\left(1-t s_{j i}\right)}{2 b}\left(\frac{(a-b c) N}{N+1}\right)^{2} .
$$

For fixed access prices, compatibility enables users to access a larger pool of developers than if platforms were not compatible. This concludes the analysis of the second period subgame.

We now solve the first period. There are two stages. Stage one is the price setting stage. Stage two is the adoption subgame where users and developers choose simultaneously which platform to join. We are interested in analyzing symmetric equilibria in which platforms set the same access prices $\left(p_{1}^{U}=p_{2}^{U}=p^{U *}\right.$ and $\left.p_{1}^{D}=p_{2}^{D}=p^{D *}\right)$.

Consider the second stage. Given $\left\{\left(p_{i}^{U}, p_{i}^{D}\right)\right\}_{i=1}^{2}$, users and developers choose which platform to join. We first study the choice by users. Because we focus on full market coverage, we have that $x_{1}=1-x_{2}$. We thus drop subscripts. In this case, the user $\widehat{j}$ indifferent between trading with platform providers 1 and 2 is at distance $x$ from 1 . Because the market is fully covered, $x$ also denotes the measure of users served by platform provider 1 and $1-x$ that of users served by platform provider 2 .

For fixed prices $p_{1}^{U}$ and $p_{2}^{U}$, the location of the indifferent user is given by:

$$
u_{\hat{j}}^{c o m}(x)-p_{1}^{U}=u_{\hat{j}}^{c o m}(1-x)-p_{2}^{U}
$$

or

$$
\frac{(1-t x)}{2 b}\left(\frac{(a-b c) N}{N+1}\right)^{2}-p_{1}^{U}=\frac{(1-t(1-x))}{2 b}\left(\frac{(a-b c) N}{N+1}\right)^{2}-p_{2}^{U} .
$$

Hence, user demand for provider 1's platform is

$$
x\left(p_{1}^{U}, p_{2}^{U}\right)=\frac{1}{2}+\frac{b\left(p_{2}^{U}-p_{1}^{U}\right)}{t(a-b c)^{2}}\left(\frac{N+1}{N}\right)^{2} .
$$


Consider now the developers. The net profit made by a developer who trades with platform provider $i \in\{1,2\}$ is

$$
\pi^{c o m}-f-p_{i}^{D}=\frac{\left(2-t+2 t x-2 t x^{2}\right)}{2 b}\left(\frac{a-b c}{N+1}\right)^{2}-f-p_{i}^{D}
$$

where we have suppressed the arguments of $x(\cdot)$ to ease notation. Developers' profits in the second period do not depend on the platform they develop for. Thus, both platforms are perceived as homogeneous by developers and they will choose platform based solely on the entry fees being charged $\left(p_{1}^{D}\right.$ and $\left.p_{2}^{D}\right)$. Bertrand competition for developers implies that the lowest entry fee will fully determine the number of active developers. ${ }^{12}$ That is, the following must be satisfied for any volume of users captured by platform provider $i$ :

$$
\frac{\left(2-t+2 t x-2 t x^{2}\right)}{2 b}\left(\frac{a-b c}{N+1}\right)^{2}-f-\min \left\{p_{1}^{D}, p_{2}^{D}\right\}=0 .
$$

Thus, given $\left\{\left(p_{i}^{U}, p_{i}^{D}\right)\right\}_{i=1}^{2}$, the equilibrium of the second-stage subgame $\left(x^{*}, N^{*}\right)$ is the solution to the system of two equations formed by (4) and (5). It is easy to see that when access prices are symmetric, $p_{1}^{U}=p_{2}^{U}$ and $p_{1}^{D}=p_{2}^{D}$, the system has one solution only and that in that solution $x^{*}=\frac{1}{2}$ (symmetry in the first stage implies symmetry in the second stage). To guarantee that $N^{*} \geq 1$, the following must happen $\frac{(a-b c)^{2}}{2 b} \geq \frac{8\left(f+\min \left\{p_{1}^{D}, p_{2}^{D}\right\}\right)}{4-t}$. We will assume that this is satisfied in equilibrium (see below). When access prices are not symmetric, the system can be shown to have at most two solutions. When there are two solutions, however, only one solution has $0 \leq x \leq 1$ and $N \geq 1.13$ Therefore, in searching for equilibria to the first-stage price choices, we need only consider the behavior of one solution to (4) and (5).

We now study the first stage where both platform providers simultaneously choose $\left\{\left(p_{i}^{U}, p_{i}^{D}\right)\right\}_{i=1}^{2}$. We begin by showing that there can be no symmetric equilibrium in which

\footnotetext{
${ }^{12}$ Although free entry implies that developers end up earning zero profits regardless of the platform they join, it is assumed that the platform charging the lowest $p^{D}$ attracts all developers, that is, in case of indifference, developers prefer the platform whose access fee is lowest.

${ }^{13}$ Moreover, the solution in the correct range is obtained continuously from $\left(x^{*}, N^{*}\right)$. Fix $p_{1}^{U}=p_{2}^{U} \equiv p^{U}$ and $p_{1}^{D}=p_{2}^{D} \equiv p^{D}$. Suppose that the parameter values are such that the only solution to the system of equations has $x^{*}=\frac{1}{2}$ and $N^{*} \geq 1$ (which is required for equilibrium). Fix a small $\epsilon>0$. For new access prices $\left\{\left(p_{i}^{U}, p_{i}^{D}\right)\right\}_{i=1}^{2}$ satisfying $p^{U}-\epsilon<p_{i}^{U}<p^{U}+\epsilon, p_{1}^{U} \neq p_{2}^{U}$ and $p^{D}-\epsilon<p_{i}^{D}<p^{D}+\epsilon$, the system has two solutions $\left(x_{1}^{*}, N_{1}^{*}\right)$ and $\left(x_{2}^{*}, N_{2}^{*}\right)$. The first solution is "close" to $\left(x^{*}, N^{*}\right)$ and satisfies $0 \leq x_{1}^{*} \leq 1$ and $N_{1}^{*} \geq 1$. The second solution is discontinuous ("far away" from $\left(x^{*}, N^{*}\right)$ ) and in the wrong range: $x_{2}^{*}>1$ and/or $N_{2}^{*}<1$.
} 
both platform providers charge an entry fee for developers above $c^{D}$. Otherwise, one of the platform providers could slightly decrease the price charged to developers, which would discontinuously increase the number of developers she serves, thereby increasing profits ${ }^{14}$ Similarly, there can be no symmetric equilibrium in which both platform providers subsidize developers $\left(p_{1}^{D}=p_{2}^{D} \equiv p^{D *}<c^{D}\right)$. Otherwise, one of the providers could unilaterally raise her entry fee, which would not affect profits made on the user side, and would stop losses on the developer side. Therefore, if a symmetric equilibrium exists, then it must be such that $p_{1}^{D}=p_{2}^{D} \equiv p^{D *}=c^{D}$.

Next, we derive a unique candidate to symmetric equilibrium for $p_{1}^{U}$ and $p_{2}^{U}$. Platform 1's profit is $\Pi_{1}\left(p_{1}^{U}, p_{2}^{U}\right)=p_{1}^{U} x\left(p_{1}^{U}, p_{2}^{U}\right)$ and platform 2 's profit is $\Pi_{2}\left(p_{1}^{U}, p_{2}^{U}\right)=p_{2}^{U}\left(1-x\left(p_{1}^{U}, p_{2}^{U}\right)\right)$, so the first-order conditions are:

$$
\begin{aligned}
x\left(p_{1}^{U}, p_{2}^{U}\right)+p_{1}^{U} \frac{\partial x\left(p_{1}^{U}\right)}{\partial p_{1}^{U}} & =0 \\
1-x\left(p_{1}^{U}, p_{2}^{U}\right)-p_{2}^{U} \frac{\partial x\left(p_{1}^{U}\right)}{\partial p_{2}^{U}} & =0 .
\end{aligned}
$$

To derive an expression for the partial derivatives, let $N(x)$ be the unique $N$ that solves (5) given $x$ :

$$
N(x)=\sqrt{\frac{\left(2-t+2 t x-2 t x^{2}\right)(a-b c)^{2}}{2 b\left(f+p_{i}^{D}\right)}}-1 .
$$

Then, using the result that $p_{1}^{D}=p_{2}^{D} \equiv p^{D *}=c^{D}$, the marginal user condition (eq. 4) can be expressed as:

$$
x\left(p_{1}^{U}, p_{2}^{U}\right)-\frac{1}{2}-\frac{b\left(p_{2}^{U}-p_{1}^{U}\right)}{t(a-b c)^{2}\left(1-\sqrt{\frac{2 b\left(f+c^{D}\right)}{\left[2-t+2 t x\left(p_{1}^{U}, p_{2}^{U}\right)-2 t\left(x\left(p_{1}^{U}, p_{2}^{U}\right)\right)^{2}\right](a-b c)^{2}}}\right)^{2}}=0 .
$$

Applying the implicit function theorem yields:

$$
\frac{\partial x\left(p_{1}^{U}, p_{2}^{U}\right)}{\partial p_{1}^{U}}=-\frac{1}{\frac{t(a-b c)^{2}\left(\frac{N(x)}{N(x)+1}\right)^{2}}{b}+\frac{2 t(1-2 x)\left(p_{2}^{U}-p_{1}^{U}\right) \sqrt{\frac{2 b\left(f+c^{D}\right)}{(a-b c)^{2}}}}{\left(2-t+2 t x-2 t x^{2}\right)^{\frac{3}{2}}\left(\frac{N(x)}{N(x)+1}\right)}}
$$

\footnotetext{
${ }^{14}$ Note as well that this would have an arbitrarily small effect on user demand for such provider's platform, which does not affect the thrust of the argument.
} 
Using this expression in the above first-order conditions, we see that the following must hold in a symmetric equilibrium:

$$
p_{1}^{U}=p_{2}^{U} \equiv p^{U *}=\frac{t(a-b c)^{2}}{2 b}\left(1-\sqrt{\frac{4 b\left(f+c^{D}\right)}{(4-t)(a-b c)^{2}}}\right)^{2} .
$$

To guarantee that $N^{*}=N\left(\frac{1}{2}\right) \geq 1$, we assume that $\frac{(a-b c)^{2}}{2 b} \geq \frac{8\left(f+c^{D}\right)}{4-t}$. This concludes our search for a candidate to symmetric equilibrium $\left(p^{U *}, p^{D *}\right)$.

To prove that both platform providers setting $\left(p^{U *}, p^{D *}\right)$ constitutes an equilibrium, it only remains to rule out deviations involving decreases in the entry fee accompanied by changes in the price charged to users ${ }^{15}$ In order to show that one of the platform providers, 1 say, has no incentive to do so, note that if 1 's entry fee is the lowest, then it will attract $n_{1}$ developers, where $n_{1}$ is given by the following free-entry condition:

$$
p_{1}^{D}=\frac{\left(2-t+2 t x-2 t x^{2}\right)}{2 b}\left(\frac{a-b c}{n_{1}+1}\right)^{2}-f .
$$

Because there is a one-to-one relationship between $p_{1}^{D}$ and $n_{1}$ (given $x$ ), we can let platform provider 1 choose $p_{1}^{U}$ and $n_{1}$ (instead of $p_{1}^{D}$ ) while keeping $p_{2}^{U}$ and $p_{2}^{D}$ fixed.

We note the following useful fact: given $p_{1}^{D}, n_{1}$ is maximal when $x=\frac{1}{2}$. Therefore, platform 1 will be able to attract $n_{1}$ developers at lowest cost when $x=\frac{1}{2} L^{16}$ When $x \neq \frac{1}{2}$, to attract $n_{1}$ developers platform 1 will need to set a lower $p_{1}^{D}$, thus earning less from this side of the market. When $x=\frac{1}{2}$, the free-entry condition (6) becomes:

$$
p_{1}^{D}=\frac{(4-t)}{4 b}\left(\frac{a-b c}{n_{1}+1}\right)^{2}-f .
$$

We show that even if the number of developers was determined by eq. (7) (this is the best case for platform 1), it will not desire to deviate. Using (4) and (7), we have that platform

\footnotetext{
${ }^{15}$ It is clear that upward changes in the entry fee do not increase profit to be made on developers and do not affect $N^{*}$, which means that no platform provider has an incentive to change its user price given that its competitor's user price is kept fixed.

${ }^{16}$ We say "cost" because the only potentially helpful deviations from $p_{1}^{D}=p_{2}^{D}=p^{D *}=c^{D}$ involve $p_{1}^{D}<c^{D}$, a negative markup.
} 
provider 1 solves

$\max _{p_{1}^{U}, n_{1}} \Pi_{1}\left(p_{1}^{U}, n_{1}\right)=\max _{p_{1}^{U}, n_{1}}\left[p_{1}^{U}\left(\frac{1}{2}+\frac{b\left(p^{U *}-p_{1}^{U}\right)}{t(a-b c)^{2}}\left(\frac{n_{1}+1}{n_{1}}\right)^{2}\right)+\frac{(4-t)(a-b c)^{2} n_{1}}{4 b\left(n_{1}+1\right)^{2}}-\left(f+c^{D}\right) n_{1}\right]$.

It is simple to show that the derivative with respect to $p_{1}^{U}$ evaluated at $\left(p^{U *}, N^{*}\right)$ is zero. In turn, the (right) derivative with respect to $n_{1}$ evaluated at $\left(p^{U *}, N^{*}\right)$ is non-positive (since $N^{*} \geq 1$ ). The question is then whether the decrease in profit from larger $n_{1}$ is compensated by the profit increase from an increase in $p_{1}^{U}$. To see that the answer is negative, we solve $\frac{\partial \Pi_{1}\left(p_{1}^{U}, n_{1}\right)}{\partial p_{1}^{U}}=0$ for $p_{1}^{U}$ to obtain

$$
p_{1}^{U}\left(n_{1}\right)=\frac{1}{4}\left(2 p^{U *}+\frac{(a-b c)^{2} n_{1}^{2} t}{b\left(n_{1}+1\right)^{2}}\right) .
$$

We then substitute $p_{1}^{U}\left(n_{1}\right)$ in $\Pi_{1}\left(p_{1}^{U}, n_{1}\right)$ to obtain profit as a function of $n_{1}$ alone, $\Pi_{1}\left(n_{1}\right)$. Finally, a little algebra shows that $\frac{\partial \Pi_{1}\left(n_{1}\right)}{\partial n_{1}}<0$ for all parameter values. Hence, both platform providers setting prices $\left(p^{U *}, p^{D *}\right)$ constitutes the unique symmetric equilibrium of the game.

The properties of such an equilibrium outcome are driven by the full evaporation of profits on the developer side because developers perceive platforms to be homogeneous. The fact that platforms are not characterized by idiosyncratic network effects implies that providers compete for users as in a traditional Hotelling framework. Hence, profits just accrue because users perceive platforms to be horizontally differentiated. In particular, equilibrium profits are equal to

$$
\Pi^{*}=\frac{t(a-b c)^{2}}{4 b}\left(\frac{N^{*}}{N^{*}+1}\right)^{2} \geq 0
$$

Note also that the marginal user achieves positive utility in equilibrium, that is,

$$
\frac{(2-3 t)(a-b c)^{2}}{4 b}\left(\frac{N^{*}}{N^{*}+1}\right)^{2}>0
$$

holds because $t \in(0,2 / 3)$.

We summarize the properties of the unique symmetric equilibrium that we have just derived as follows.

Proposition 1 Suppose that there exist two platform providers that sell compatible plat- 
forms. The unique symmetric equilibrium yields profits for each firm equal to

$$
\Pi^{*}=\frac{t(a-b c)^{2}}{4 b}\left(\frac{N^{*}}{N^{*}+1}\right)^{2} .
$$

Moreover, users are not subsidized to purchase the platform, whereas developers are charged an entry fee equal to marginal cost: $p^{U *} \geq 0=c^{U}$ and $p^{D *}=c^{D}$.

\subsection{Social efficiency}

We now derive the welfare-maximizing number of developers under the assumption that the social planner can control the number of developers who become active in the first period, but not their subsequent behavior. We show that price competition for developers is too harsh and results in excessive entry.

In this second-best scenario, a welfare-maximizing social planner who targeted full market coverage would face the following problem:

$$
\begin{aligned}
\max _{N} W^{c o m}(N) & =\max _{N}[2 \underbrace{\int_{0}^{\frac{1}{2}} \frac{(1-t j)(a-b c)^{2}}{2 b}\left(\frac{N}{N+1}\right)^{2} d j}_{\text {Users' surplus }}+\underbrace{\left.\left(\frac{(4-t)(a-b c)^{2}}{4 b}\left(\frac{1}{N+1}\right)^{2}-f-c^{D}\right) N\right]}_{\text {Platforms' profits }} \\
& =\max _{N}\left[\frac{(4-t)(a-b c)^{2}}{8 b}\left(1-\frac{1}{(N+1)^{2}}\right)-\left(f+c^{D}\right) N\right] .
\end{aligned}
$$

It is straightforward to show that the efficient number of developers is given by the solution to the first-order condition:

$$
N^{e}=\left(\frac{(4-t)(a-b c)^{2}}{4 b\left(f+c^{D}\right)}\right)^{\frac{1}{3}}-1
$$

Hence, the fact that $N^{*}=\left(\frac{(4-t)(a-b c)^{2}}{4 b\left(f+c^{D}\right)}\right)^{\frac{1}{2}}-1$ and the assumption that $\frac{(a-b c)^{2}}{2 b} \geq$ $\frac{8\left(f+c^{D}\right)}{4-t}$ (made earlier to ensure that $N^{*} \geq 1$ ) yield that $N^{e}<N^{*}$. Using equation 9 , it is straightforward that the entry fee $p^{D e}$ that implements the socially efficient outcome exceeds marginal cost $c^{D}$. The result that we have just proved is stated in the following proposition. 
Proposition 2 Suppose there exist two competing platform providers that sell compatible platforms. Then entry by developers is excessive from a social planner's viewpoint: $N^{*}>N^{e}$. Moreover, the socially efficient entry fee does not subsidize developers: $p^{D e}>c^{D}$.

Bertrand competition for developers implies that the social marginal cost of promoting developer entry coincides with the entry costs faced by an extra developer. Therefore, both the additional developer and a social planner would consider the exact same costs of entry, $f+c^{D}$. However, the additional developer and the social planner differ in their assessment of the benefits of entry. The second-period profits that an extra developer earns if it enters are always greater than the additional second-period social surplus generated by this extra developer. Hence, entry is excessive from a social welfare standpoint, since the social incentives to promote developer entry are weaker than the private incentives to enter.

\section{Incompatible platforms}

When platforms are incompatible, the $n_{i}$ application developers who have traded with platform provider $i$ develop applications that work exclusively with that platform.

We begin by solving for the second period outcomes, given $\left\{\left(p_{i}^{U}, p_{i}^{D} ; x_{i}, n_{i}\right)\right\}_{i=1}^{2}$. Amongst users who bought platform $i \in\{1,2\}$, let $x_{i}$ denote the distance from $i$ for the individual located farthest away. Monotonicity implies that those individuals whose distance from $i$ is less than $x_{i}$ must have traded with $i$ as well, so the measure of users served by platform provider $i$ is $x_{i}$. If the $n_{i}$ developers who have traded with platform $i$ charge price $\rho_{i}$, then the demand for applications by user $j$ located at distance $s_{j i} \in\left[0, x_{i}\right]$ from platform $i$ is given by $q_{j}\left(\rho_{i}, s_{j i}\right)=\left(1-t s_{j i}\right)\left(a-b \rho_{i}\right)$. It follows that aggregate demand for applications functional on $i$ equals $Q_{i}\left(\rho_{i}, x_{i}\right)=\int_{0}^{x_{i}} q_{j}\left(\rho_{i}, s_{j i}\right) d j=x_{i}\left(1-\frac{t x_{i}}{2}\right)\left(a-b \rho_{i}\right)$, and hence inverse demand is

$$
\rho_{i}\left(Q_{i}, x_{i}\right)=\frac{a}{b}-\frac{2 Q_{i}}{b x_{i}\left(2-t x_{i}\right)} .
$$

Having obtained aggregate demand, it is standard to show that $\rho_{i}^{i n c}=\frac{a+n_{i} b c}{b\left(n_{i}+1\right)}$ is the second-period equilibrium price of platform $i$ 's applications under Cournot competition, and hence each earns the following post-entry profits:

$$
\pi_{i}^{i n c}=\frac{x_{i}\left(2-t x_{i}\right)}{2 b}\left(\frac{a-b c}{n_{i}+1}\right)^{2} .
$$


User $j$ who is located at distance $s_{j i}$ from platform $i \in\left[0, x_{i}\right]$ and who has acquired such platform, attains the following second-period surplus:

$$
u_{j}^{i n c}\left(s_{j i}\right)=\int_{\rho_{i}^{i n c}}^{a / b}\left(1-t s_{j i}\right)\left(a-b \rho_{i}\right) d \rho_{i}=\frac{\left(1-t s_{j i}\right)\left(a-b \rho_{i}^{i n c}\right)^{2}}{2 b}=\frac{\left(1-t s_{j i}\right) n_{i}^{2}}{2 b}\left(\frac{a-b c}{n_{i}+1}\right)^{2} .
$$

Observe that, ceteris paribus, users would benefit from competition between developers, whereas a larger market size in the second period would make developers better off. Hence, both sides exhibit positive inter-group network effects that must be acknowledged by platforms when setting access prices in the first period. In addition, the developer side exhibits negative intra-group network effects, since, for a fixed measure of users, increasing the number of developers would destroy second-period profits. This concludes the analysis of the second period subgame.

We now solve the first-period. We are interested in analyzing symmetric equilibria in which platforms set the same access prices $\left(p_{1}^{U}=p_{2}^{U}=p^{U * *}\right.$ and $\left.p_{1}^{D}=p_{2}^{D}=p^{D * *}\right)$. Consider first the second stage. Given $\left\{\left(p_{i}^{U}, p_{i}^{D}\right)\right\}_{i=1}^{2}$, users and developers choose which platform to join. We first study the choice by users. Because $x_{1}=1-x_{2}$ (i.e., the market is fully covered), we drop subscripts so that $x$ denotes the measure of users served by platform provider 1 and $1-x$ denotes the measure of users served by platform provider 2 . The location of the marginal user $\widehat{j}$ given that her distance from platform 1 is $x$ must be given by $u_{\hat{j}}^{i n c}(x)-p_{1}^{U}=u_{\hat{j}}^{i n c}(1-x)-p_{2}^{U}$, or

$$
\frac{(1-t x) n_{1}^{2}}{2 b}\left(\frac{a-b c}{n_{1}+1}\right)^{2}-p_{1}^{U}=\frac{(1-t(1-x)) n_{2}^{2}}{2 b}\left(\frac{a-b c}{n_{2}+1}\right)^{2}-p_{2}^{U} .
$$

Regarding the other side of the market, the following free-entry condition must hold for those developers trading with platform provider $i \in\{1,2\}$ :

$$
p_{i}^{D}=\frac{x_{i}\left(2-t x_{i}\right)}{2 b}\left(\frac{a-b c}{n_{i}+1}\right)^{2}-f,
$$

where $x_{i}=x$ if $i=1$ and $x_{i}=1-x$ otherwise.

Given $\left\{\left(p_{i}^{U}, p_{i}^{D}\right)\right\}_{i=1}^{2}$, the outcome to the second-stage subgame $\left(x, n_{1}\right.$, and $\left.n_{2}\right)$ is the solution to the system of three equations and three unknowns formed by 12 and 13 , $i=1,2)$. Numerical analysis shows that there may be more than one solution to that 
system 17 Different solutions correspond to different ways in which users and developers coordinate their adoption decisions of which platform to join. For example, when access prices are symmetric $p_{1}^{U}=p_{2}^{U}$ and $p_{1}^{D}=p_{2}^{D}$, depending on the parameter values, there is either a unique solution $\left(x^{* *}, N^{* *}\right.$ ) (this solution satisfies $x^{* *}=\frac{1}{2}$ ) or there are three solutions $\left(x_{1}^{* *}, N_{1}^{* *}\right),\left(x_{2}^{* *}, N_{2}^{* *}\right)$, and $\left(x_{3}^{* *}, N_{3}^{* *}\right)$. One of the three solutions, say $\left(x_{2}^{* *}, N_{2}^{* *}\right)$, has $x_{2}^{* *}=\frac{1}{2}$. Another solution, say $\left(x_{1}^{* *}, N_{1}^{* *}\right)$, has $x_{1}^{* *}<\frac{1}{2}$. The other solution has $x_{3}^{* *}>\frac{1}{2}$.

We deal with the issue of multiple solutions to the system by requiring symmetric equilibria to meet a monotonicity criterion. Following Caillaud and Jullien (2003), we will require that unilateral deviations by one platform that involve (weakly) increasing the access prices should not lead to increases in user and developer demand for the platform 18 Only the solution with $x_{2}^{* *}=\frac{1}{2}$ satisfies this criterion. Thus, in searching for equilibria when access prices are symmetric, we will consider the solution with $x_{2}^{* *}=\frac{1}{2}$ only. When access prices are asymmetric, the system has three solutions or no solutions at all. When there are three solutions, only one solution satisfies Caillaud and Jullien's (2003) monotonicity criterion ${ }^{19}$

We now derive the equilibrium access prices. Because the free-entry condition (13) implies that

$$
\left(\frac{(a-b c) n_{i}}{n_{i}+1}\right)^{2}=\left(a-b c-\sqrt{\frac{2 b\left(p_{i}^{D}+f\right)}{x_{i}\left(2-t x_{i}\right)}}\right)^{2}
$$

expression 12 can be rewritten as:

$$
\frac{(1-t x)\left(a-b c-\sqrt{\frac{2 b\left(p_{1}^{D}+f\right)}{x(2-t x)}}\right)^{2}}{2 b}+\frac{(t-1-t x)\left(a-b c-\sqrt{\frac{2 b\left(p_{2}^{D}+f\right)}{(1-x)(2-t(1-x))}}\right)^{2}}{2 b}+p_{2}^{U}-p_{1}^{U}=0 .
$$

This equation implicitly defines an expression for $x$ as a function of $p_{1}^{U}$ and $p_{1}^{D}$ (that is, we have that $x\left(p_{1}^{U}, p_{1}^{D}\right)$, although we will sometimes suppress the dependence to save space).

\footnotetext{
${ }^{17}$ The Mathematica code is available from the authors.

${ }^{18}$ The monotonicity criterion is (trivially) satisfied in the unique symmetric equilibrium under compatibility derived in Section 3.

${ }^{19}$ Moreover, the only solution that satisfies Caillaud and Jullien's criterion is obtained continuously from $\left(x_{2}^{* *}, N_{2}^{* *}\right)$. Fix $p_{1}^{U}=p_{2}^{U} \equiv p^{U}$ and $p_{1}^{D}=p_{2}^{D} \equiv p^{D}$. Consider the solution $\left(x_{2}^{* *}, N_{2}^{* *}\right)$ with $x_{2}^{* *}=\frac{1}{2}$. Fix a small $\epsilon>0$. For new access prices $\left\{\left(p_{i}^{U}, p_{i}^{D}\right)\right\}_{i=1}^{2}$ satisfying $p^{U}-\epsilon<p_{i}^{U}<p^{U}+\epsilon$ and $p^{D}-\epsilon<p_{i}^{D}<p^{D}+\epsilon$, the system has three solutions $\left(\hat{x}_{1}^{* *}, \hat{N}_{1}^{* *}\right),\left(\hat{x}_{2}^{* *}, \hat{N}_{2}^{* *}\right)$, and $\left(\hat{x}_{3}^{* *}, \hat{N}_{3}^{* *}\right)$. The first solution is "close" to $\left(x_{1}^{* *}, N_{1}^{* *}\right)$, the second to $\left(x_{2}^{* *}, N_{2}^{* *}\right)$, and the third to $\left(x_{3}^{* *}, N_{3}^{* *}\right)$. Only $\left(\hat{x}_{2}^{* *}, \hat{N}_{2}^{* *}\right)$ satisfies the monotonicity criterion.
} 
Using (13) and (14), platform provider 1's problem can be written as follows:

$$
\begin{aligned}
\max _{p_{1}^{U}, p_{1}^{D}} \Pi_{1}\left(p_{1}^{U}, p_{1}^{D}\right) & =\max _{p_{1}^{U}, p_{1}^{D}}\left[p_{1}^{U} x\left(p_{1}^{U}, p_{1}^{D}\right)+\left(p_{1}^{D}-c^{D}\right) n_{1}\left(p_{1}^{U}, p_{1}^{D}\right)\right] \\
& =\max _{p_{1}^{U}, p_{1}^{D}}\left[p_{1}^{U} x\left(p_{1}^{U}, p_{1}^{D}\right)+\left(p_{1}^{D}-c^{D}\right)\left(\frac{(a-b c) \sqrt{x\left(p_{1}^{U}, p_{1}^{D}\right)\left(2-t x\left(p_{1}^{U}, p_{1}^{D}\right)\right)}}{\sqrt{2 b\left(p_{1}^{D}+f\right)}}-1\right)\right] .
\end{aligned}
$$

Developer surplus is fully extracted via $p_{1}^{D}$ (since all developers are symmetric) and user surplus is extracted via $p_{1}^{U}$, although all users enjoy a strictly positive utility because of full market coverage. Note that user surplus can be captured either directly or via the developers.

The first-order conditions are:

$$
x\left(p_{1}^{U}, p_{1}^{D}\right)+\left(p_{1}^{U}+\frac{\left(p_{1}^{D}-c^{D}\right)(a-b c)\left(1-t x\left(p_{1}^{U}, p_{1}^{D}\right)\right)}{\sqrt{2 b\left(p_{1}^{D}+f\right) x\left(p_{1}^{U}, p_{1}^{D}\right)\left(2-t x\left(p_{1}^{U}, p_{1}^{D}\right)\right)}}\right) \frac{\partial x\left(p_{1}^{U}, p_{1}^{D}\right)}{\partial p_{1}^{U}}=0
$$

and

$$
\begin{gathered}
p_{1}^{U} \frac{\partial x\left(p_{1}^{U}, p_{1}^{D}\right)}{\partial p_{1}^{D}}+\frac{(a-b c) \sqrt{x\left(p_{1}^{U}, p_{1}^{D}\right)\left(2-t x\left(p_{1}^{U}, p_{1}^{D}\right)\right)}}{\sqrt{2 b\left(p_{1}^{D}+f\right)}}-1+\left(p_{1}^{D}-c^{D}\right) \times \\
\left(\frac{(a-b c)\left(1-t x\left(p_{1}^{U}, p_{1}^{D}\right)\right)}{\sqrt{2 b\left(p_{1}^{D}+f\right) x\left(p_{1}^{U}, p_{1}^{D}\right)\left(2-t x\left(p_{1}^{U}, p_{1}^{D}\right)\right)}} \frac{\partial x\left(p_{1}^{U}, p_{1}^{D}\right)}{\partial p_{1}^{D}}-\frac{(a-b c) \sqrt{x\left(p_{1}^{U}, p_{1}^{D}\right)\left(2-t x\left(p_{1}^{U}, p_{1}^{D}\right)\right)}}{2 \sqrt{2 b\left(p_{1}^{D}+f\right)^{3}}}\right)=0
\end{gathered}
$$

Note from expression (15) that there is an additional term relative to traditional settings without network effects owing to the inter-group network effect exerted by users on developers. The second term within the parenthesis reflects the fact that changing the user price affects the number of users who choose to acquire the platform, which in turn affects the developers' demand for the platform and hence the gain/loss made on the developer side. A similar observation can be made regarding (16), taking into account that changing the entry fee affects the number of developers not only by varying the (sunk) cost of entry but also by changing the size of the second-period market.

Given the algebraic complexity of solving for the symmetric equilibrium under incompatibility, we relegate that derivation to the Appendix (see proof of Proposition 3). Similarly to the case of compatible platforms, we use the implicit function theorem to derive expressions for $\partial x\left(p_{1}^{U}, p_{1}^{D}\right) / \partial p_{1}^{U}$ and $\partial x\left(p_{1}^{U}, p_{1}^{D}\right) / \partial p_{1}^{D}$. We use those expressions in conjunction to equations (15) and (16) and the assumption of symmetry to solve for the equilibrim access prices $p^{U * *}$ and $p^{D * *}$. 
To simplify notation, let

$$
Z \equiv \frac{4\left(f+c^{D}\right)\left(t^{2}-6 t+12\right)^{3}}{(4-t)^{2} t^{3}\left(4+2 t-t^{2}\right)} \quad \text { and } \quad Z^{\prime} \equiv \frac{4\left(f+c^{D}\right)\left(32+8 t^{2}-24 t-t^{3}\right)^{3}}{t^{3}\left(8-t^{2}\right)(4-t)^{5}}
$$

and note that $Z^{\prime}<Z$ for $0<t<2 / 3$ (see Fig. 2). The following proposition establishes that $\frac{(a-b c)^{2}}{2 b} \geq Z^{\prime}$ is a necessary and sufficient condition for a symmetric equilibrium satisfying Caillaud and Jullien's (2003) monotonicity criterion to exist under incompatibility. We also show that the unique symmetric equilibrium exhibits the property that developers are never subsidized, whereas users may or may not be subsidized depending on the extent of platform differentiation.

Proposition 3 Suppose there exist two competing platform providers that sell incompatible platforms. A symmetric equilibrium satisfying the monotonicity criterion exists if and only if $\frac{(a-b c)^{2}}{2 b} \geq Z^{\prime}$. In the unique symmetric equilibrium developers are never subsidized, and users are subsidized if and only if $\frac{(a-b c)^{2}}{2 b}<Z$. In particular, it holds that $p^{U * *}<0=c^{U}$ and $p^{D * *}>c^{D}$ for $\frac{(a-b c)^{2}}{2 b}<Z$, whereas $p^{U * *} \geq 0=c^{U}$ and $p^{D * *}>c^{D}$ for $\frac{(a-b c)^{2}}{2 b} \geq Z$.

Proof. See Appendix.

Because our model has infinitely many potential developers, incompatible platforms do not compete to attract them via access prices but rather via the size of the user network. When horizontal differentiation is weak, platform providers compete fiercely for users by subsidizing their access to the platform with the aim of boosting developer entry and thus profits made on the developer side (as stems from (15) taking into account that $p^{D}>c^{D}$ and $\partial x\left(p_{1}^{U}, p_{1}^{D}\right) / \partial p_{1}^{U}<0$ at the symmetric equilibrium prices).

Incompatibility allows platforms to vertically differentiate through the number of applications that they offer. However, since in this section we are studying symmetric equilibria, the number of developers is the same for both platforms. This implies that, in equilibrium, the products end up being horizontally differentiated only. In Section 5 we consider asymmetric equilibria and show that endogenous vertical differences arise on the grounds of the quantity of applications offered on each platform.

We have studied symmetric equilibria under the assumption that whenever $p_{1}^{U}=p_{2}^{U}$ and $p_{1}^{D}=p_{2}^{D}$ the equilibrium to the second-stage subgame has $x=\frac{1}{2}$ and $n_{1}=n_{2}$. However, as mentioned above, there may be other solutions to the system $(12)$ and $(13, i=1,2)$ at 
the equilibrium access prices. Remark 1 says that whenever the parameter values are such that the solution $x=\frac{1}{2}$ satisfies Caillaud and Jullien's (2003) monotonicity criterion, there are three equilibria of the second-stage subgame: $(i)$ the symmetric solution $\left(x=\frac{1}{2}\right.$ and $\left.n_{1}=n_{2}\right)$, (ii) an asymmetric solution with market dominance by platform $2\left(x^{\prime}<\frac{1}{2}\right.$ and $\left.n_{1}^{\prime}<n_{2}^{\prime}\right)$; and $(i i i)$ an asymmetric solution with market dominance by platform $1\left(x^{\prime \prime}=1-x^{\prime}\right.$ and $n_{1}^{\prime \prime}=n_{2}^{\prime}>n_{2}^{\prime \prime}=n_{1}^{\prime}$ ). (See Figure 2 below.)

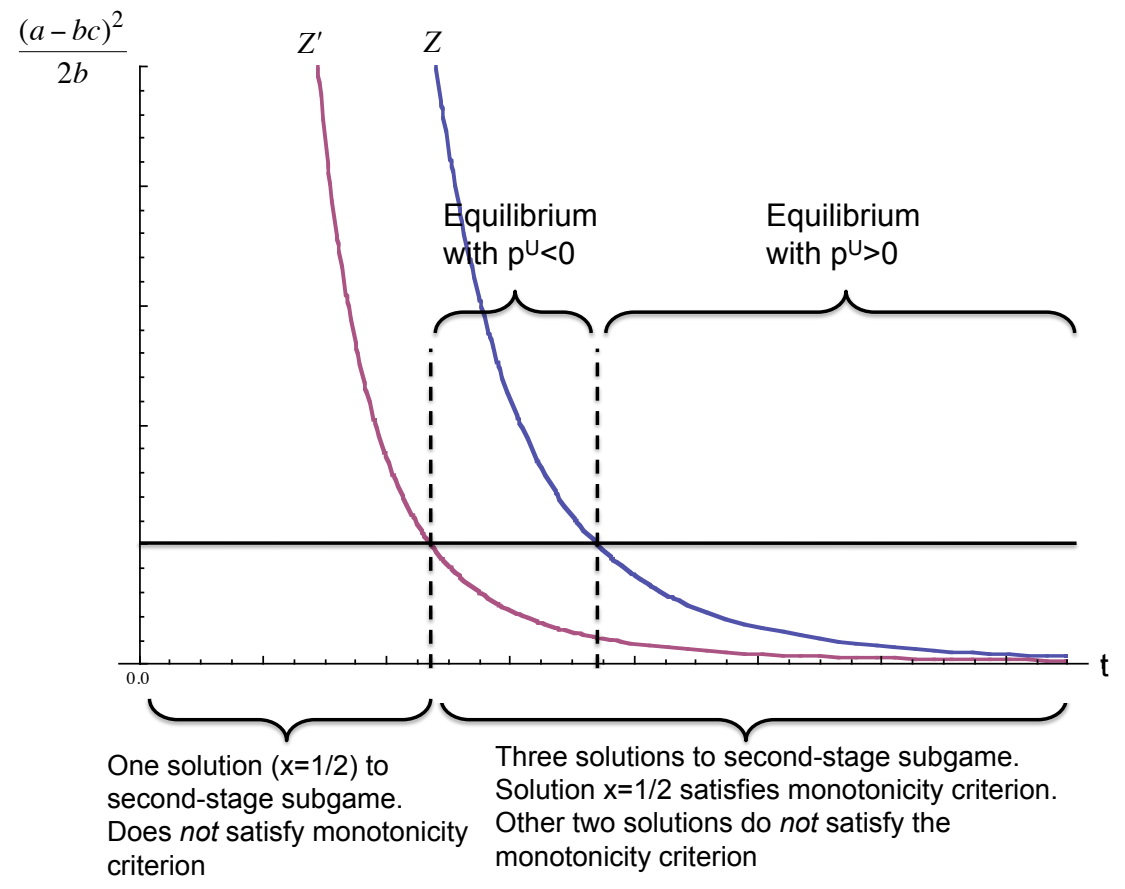

Figure 2: Solutions to second-stage subgame.

Remark 1 Suppose there exist two competing platform providers that sell incompatible platforms at the equilibrium prices derived in Proposition 3. Then, the platform adoption subgame (the second stage of the first period, see Figure 1) has three equilibria: $\left(x=\frac{1}{2}, n_{1}=n_{2}\right)$; $\left(x^{\prime}<\frac{1}{2}, n_{1}^{\prime}<n_{2}^{\prime}\right)$; and $\left(x^{\prime \prime}=1-x^{\prime}, n_{1}^{\prime \prime}=n_{2}^{\prime}>n_{2}^{\prime \prime}=n_{1}^{\prime}\right)$ if and only if $\frac{(a-b c)^{2}}{2 b} \geq Z^{\prime}$. Otherwise, there is one equilibrium only: $\left(x=\frac{1}{2}, n_{1}=n_{2}\right)$.

Proof. See Appendix.

We end by pointing out that numerical simulations show that the two solutions with $x \neq \frac{1}{2}$ do not satisfy the monotonicity refinement and thus we ignore them ${ }^{20}$ Figure 2 illustrates the result.

\footnotetext{
${ }^{20}$ The Mathematica code is available from the authors.
} 


\subsection{Social efficiency}

A social planner which targeted all users and chose to let both platform providers operate would solve:21

$$
\begin{aligned}
\max _{n} W^{i n c}(n) & =\max _{n} 2[\underbrace{\int_{0}^{\frac{1}{2}} \frac{(1-t j) n^{2}}{2 b}\left(\frac{a-b c}{n+1}\right)^{2} d j}_{\text {Users' surplus }}+\underbrace{\left(\frac{4-t}{8 b}\left(\frac{a-b c}{n+1}\right)^{2}-f-c^{D}\right) n}_{\text {Platforms' profits }}] \\
& =\max _{n}\left[\frac{(4-t)(a-b c)^{2}}{8 b}\left(1-\frac{1}{(n+1)^{2}}\right)-2\left(f+c^{D}\right) n\right] .
\end{aligned}
$$

Therefore,

$$
n^{e}=\left(\frac{(4-t)(a-b c)^{2}}{8 b\left(f+c^{D}\right)}\right)^{\frac{1}{3}}-1 \quad \text { and } \quad p^{D e}=\left(\frac{(4-t)(a-b c)^{2}\left(f+c^{D}\right)^{2}}{8 b}\right)^{\frac{1}{3}}-f
$$

The following proposition shows that (symmetric) competition between incompatible platforms leads to insufficient entry from a social efficiency point of view. Providers of incompatible platforms do not internalize all the positive effects of the network effect exerted on users by developers because they cannot appropriate all the gains from trade that accrue to users. As a result, platform providers promote less than socially desirable entry by developers, which results in social welfare losses because of insufficient consumption of applications.

Proposition 4 Suppose there exist two competing platform providers that sell incompatible platforms. Then the symmetric outcome of duopolistic competition is such that entry by developers is insufficient from a social planner's viewpoint: $n^{* *}<n^{e}$. Moreover, the socially efficient entry fee does not subsidize developers: $p^{D e}>c^{D}$.

Proof. See Appendix.

\subsubsection{Welfare comparison: compatible vs. incompatible platforms}

We end this section by comparing social welfare. When a social planner can control the number of developers who become active in the first period but not their subsequent behavior

\footnotetext{
${ }^{21}$ We should point out that a social planner may prefer having just one platform provider serving both sides of the market so as to avoid underexploitation of network effects and duplication of costs.
} 
in the second period, welfare under compatibility and incompatibility are, respectively:

$$
\max _{N} W^{c o m}(N)=\max _{N}\left[\frac{(4-t)(a-b c)^{2}}{8 b}\left(1-\frac{1}{(N+1)^{2}}\right)-\left(f+c^{D}\right) N\right]
$$

and

$$
\max _{n} W^{i n c}(n)=\max _{n}\left[\frac{(4-t)(a-b c)^{2}}{8 b}\left(1-\frac{1}{(n+1)^{2}}\right)-2\left(f+c^{D}\right) n\right] .
$$

Direct inspection and a revealed preference argument imply that compatibility leads to higher total welfare. The reason is that in order for both intellectual property regimes to produce a given level of user and developer surplus (exclusive of entry costs), incompatibility requires duplication of $\operatorname{costs}\left(f+c^{D}\right.$ vs. $\left.2\left(f+c^{D}\right)\right)$ and thus compatibility is preferred. This is due to the underexploitation of network effects that occurs when platforms are incompatible (as applications are platform-specific in this case).

\section{$5 \quad$ Platform compatibility versus incompatibility}

Many industries with two-sided platforms are characterized by platform incompatibility and strong market dominance by one single firm. The purpose of this section is to investigate why this may be so partly in light of our previous results. A natural starting point is to compare the properties of symmetric equilibria depending on whether platforms are compatible or not. More specifically, we examine which industry structure leads to highest equilibrium profits. To get a sense, we consider the case in which the only fixed cost incurred by a developer is the entry fee paid to access a platform (i.e., $f=c^{D}=0$ ). In this scenario, it is easy to prove that $p^{D * *}=\frac{t^{2}(4-t)(a-b c)^{2}}{128 b}$. Using this result, it is a matter of simple algebra to show that

$$
p^{U *}=\frac{t(a-b c)^{2}}{2 b}>p^{U * *}=\frac{t\left(4+2 t-t^{2}\right)(a-b c)^{2}}{32 b}>0
$$

and

$$
\Pi^{*}=\frac{t(a-b c)^{2}}{4 b}>\Pi^{* *}=\frac{t\left(24-t^{2}-4 t\right)(a-b c)^{2}}{128 b}>0 .
$$

This suggests that the (symmetric equilibrium) prices charged to users and profits are greater under platform compatibility than under incompatibility. ${ }^{22}$ This raises the question

\footnotetext{
${ }^{22}$ We have also performed many numerical simulations with $f+c^{D}>0$, and we have found no counterexample to this claim. See Figure 3. The Mathematica code is available from the authors.
} 
of why platform providers do not somehow negotiate to make their platforms compatible, which is also the socially efficient outcome because it avoids cost duplication (see Subsection 4.1.1). One plausible answer is that there might exist other equilibria when platforms are incompatible in which (at least) one of the platform providers earns more profits than in any equilibrium that arises when platforms are compatible.

We now proceed to investigate whether there are asymmetric equilibria under incompatibility that may be preferred by (at least) one platform provider over all equilibria in the compatible case. We first consider the case of homogenous users $(t=0)$ and then extend the analysis to the less tractable case of heterogeneous users $(0<t<2 / 3)$. We will find that the only equilibrium under compatibility is the symmetric equilibrium derived in Section 3 , and that asymmetric equilibria with one platform cornering the market exist in the case of incompatible platforms. Moreover, market dominance by an incompatible platform leads to higher profits than compatibility when horizontal differences between platforms $t$ are low.

Note that the importance of studying asymmetric equilibria is reinforced by the fact that in the incompatible case, symmetric equilibria do not exist when $t$ is low. In particular,

the requirement that $\frac{(a-b c)^{2}}{2 b} \geq Z^{\prime}$ for a symmetric equilibrium to exist (see Proposition 3) becomes unrealistically severe as $t \downarrow 0$, since the right hand side of the inequality grows without bound as $t$ decreases (for positive $f+c^{D}$ ). This suggests that asymmetric equilibria with a dominant platform may be the proper benchmark when making comparisons between incompatibility and compatibility when $t$ is low.

\subsection{Homogeneous users $(t=0)$}

\subsubsection{Asymmetric equilibria when platforms are compatible}

We begin with a general development that will also be helpful for the case $t>0$ (covered in Section 5.2) and later specialize it to $t=0$.

An asymmetric equilibrium under compatibility must involve platform providers charging different prices to developers. This implies that one of the platforms must be making a loss on the developer side (otherwise, the rival would undercut it). Suppose, without loss of generality, that platform 1 is charging $p_{1}^{D}<c^{D}$ and $p_{1}^{D}<p_{2}^{D}$. In this case, platform 2's profits are

$$
\Pi_{2}\left(p_{2}^{U}\right)=p_{2}^{U}\left(1-x\left(p_{1}^{U}, p_{2}^{U}\right)\right)
$$


where, using (4) and (5), $x\left(p_{1}^{U}, p_{2}^{U}\right)$ is implicitly given by:

$$
x\left(p_{1}^{U}, p_{2}^{U}\right)-\frac{1}{2}-\frac{b\left(p_{2}^{U}-p_{1}^{U}\right)}{t(a-b c)^{2}\left(1-\sqrt{\left.\frac{2 b\left(f+p_{1}^{D}\right)}{\left[2-t+2 t x\left(p_{1}^{U}, p_{2}^{U}\right)-2 t\left(x\left(p_{1}^{U}, p_{2}^{U}\right)\right)^{2}\right](a-b c)^{2}}\right)^{2}}\right.}=0
$$

In turn, platform 1's profits are

$\Pi_{1}\left(p_{1}^{U}, p_{1}^{D}\right)=p_{1}^{U} x\left(p_{1}^{U}, p_{2}^{U}\right)+\left(p_{1}^{D}-c^{D}\right)\left(\sqrt{\frac{\left[2-t+2 t x\left(p_{1}^{U}, p_{2}^{U}\right)-2 t\left(x\left(p_{1}^{U}, p_{2}^{U}\right)\right)^{2}\right](a-b c)^{2}}{2 b\left(f+p_{1}^{D}\right)}}-1\right)$.

For simplicity, we will drop the arguments of $x\left(p_{1}^{U}, p_{2}^{U}\right)$, so that the free-entry condition can be written as

$$
N=\sqrt{\frac{\left(2-t+2 t x-2 t x^{2}\right)(a-b c)^{2}}{2 b\left(f+p_{1}^{D}\right)}}-1,
$$

and the marginal user condition can be written as

$$
x=\frac{1}{2}+\frac{b\left(p_{2}^{U}-p_{1}^{U}\right)}{t(a-b c)^{2}}\left(\frac{N+1}{N}\right)^{2}
$$

with

$$
\begin{aligned}
\frac{\partial x\left(p_{1}^{U}, p_{2}^{U}\right)}{\partial p_{1}^{U}} & =-\frac{1}{\frac{t(a-b c)^{2} N^{2}}{b(N+1)^{2}}+\frac{2 t(a-b c)^{2}(1-2 x)\left(p_{2}^{U}-p_{1}^{U}\right)}{2 b\left(f+p_{1}^{D}\right)(N+1)^{2} N}} \\
\frac{\partial x\left(p_{1}^{U}, p_{2}^{U}\right)}{\partial p_{1}^{D}} & =\frac{b\left(p_{2}^{U}-p_{1}^{U}\right)(N+1)^{2}}{t(a-b c)^{2}\left(f+p_{1}^{D}\right) N^{3}+t(a-b c)^{2}(1-2 x)\left(p_{2}^{U}-p_{1}^{U}\right)}
\end{aligned}
$$

After some manipulations and noting that $\partial x\left(p_{1}^{U}, p_{2}^{U}\right) / \partial p_{2}^{U}=-\partial x\left(p_{1}^{U}, p_{2}^{U}\right) / \partial p_{1}^{U}$, the firstorder conditions for $p_{2}^{U}, p_{1}^{U}$, and $p_{1}^{D}$ can be written, respectively, as:

$$
\begin{gathered}
0=(1-x)\left(\frac{t(a-b c)^{2} N^{2}}{b(N+1)^{2}}+\frac{2 t(a-b c)^{2}(1-2 x)\left(p_{2}^{U}-p_{1}^{U}\right)}{2 b\left(f+p_{1}^{D}\right)(N+1)^{2} N}\right)-p_{2}^{U}, \\
0=x\left(\frac{t(a-b c)^{2} N^{2}}{b(N+1)^{2}}+\frac{2 t(a-b c)^{2}(1-2 x)\left(p_{2}^{U}-p_{1}^{U}\right)}{2 b\left(f+p_{1}^{D}\right)(N+1)^{2} N}\right)-\left(p_{1}^{U}+\frac{t(a-b c)^{2}(1-2 x)\left(p_{1}^{D}-c^{D}\right)}{2 b(N+1)\left(f+p_{1}^{D}\right)}\right),
\end{gathered}
$$




$$
\begin{aligned}
0= & \left(p_{1}^{U}+\frac{t(a-b c)^{2}(1-2 x)\left(p_{1}^{D}-c^{D}\right)}{2 b(N+1)\left(f+p_{1}^{D}\right)}\right) b\left(p_{2}^{U}-p_{1}^{U}\right)(N+1)^{2}+ \\
& {\left[t(a-b c)^{2}\left(f+p_{1}^{D}\right) N^{3}+t(a-b c)^{2}(1-2 x)\left(p_{2}^{U}-p_{1}^{U}\right)\right]\left(N-\frac{\left(p_{1}^{D}-c^{D}\right)(N+1)}{2\left(f+p_{1}^{D}\right)}\right) . }
\end{aligned}
$$

Thus, a necessary condition for an asymmetric equilibrium $\left(p_{1}^{U}, p_{1}^{D}, p_{2}^{U}, p_{2}^{D}, x\right.$, and $\left.N\right)$ with $p_{1}^{D}<c^{D}$ and $p_{1}^{D}<p_{2}^{D}$ to exist is that the system of five equations formed by (17)-(21) be satisfied.

To see that there is no asymmetric equilibrium, note that when $t=0$, equations $(19)$ and (20) become $0=p_{2}^{U}$ and $0=p_{1}^{U}$. Therefore, platform provider 1 would prefer to raise $p_{1}^{D}$ (since the right hand side of (21) would be always positive). We conclude that when $t=0$ the unique equilibrium of the game is the symmetric equilibrium derived in Section 3 .

\subsubsection{Asymmetric equilibria when platforms are incompatible}

When $t=0$, compatibility yields zero profits for both providers. We now show that there exists an equilibrium under incompatibility in which one platform provider dominates the market and makes positive profits. The importance of asymmetric equilibria has already been highlighted by Katz and Shapiro's (1985) classical work on one-sided platforms, and more recently, by Caillaud and Jullien (2003) in a setting in which both sides of the platform bargain efficiently, unlike ours in which second-period trade efficiency depends on the platform providers' first-period pricing structures.

Let $\left\{p_{i}^{U}, p_{i}^{D}\right\}_{i=1}^{2}$ denote a candidate equilibrium strategy profile such that platform provider 1 corners the market. In this case, not only should users derive positive utility by purchasing platform 1 , but they should also prefer trading with 1 rather than 2 . Hence, we must have that

$$
\frac{n_{1}^{2}}{2 b}\left(\frac{a-b c}{n_{1}+1}\right)^{2}-p_{1}^{U} \geq-p_{2}^{U}
$$

where $n_{1}$ is given by the free-entry condition: $p_{1}^{D}=\frac{1}{b}\left(\frac{a-b c}{n_{1}+1}\right)^{2}-f$.

We focus throughout on the most optimistic expectations for the dominant platform (i.e., platform 1). Thus, upon observing any pair of access prices charged by a platform, it holds that a user expects all others to join platform 1 unless $p_{2}^{U} \leq \widetilde{p}_{2}^{U}=p_{1}^{U}-\frac{n_{1}}{2 b}\left(\frac{a-b c}{n_{1}+1}\right)^{2}$, in which case they all coordinate on platform 2 (since getting access to 1 is dominated). We study the most profitable deviation that platform provider 2 could undertake. In order to avoid infinite losses that would ensue from trying to attract developers, it can only deviate 
by setting a price for users such that it attracts them all. This can be done by charging a price (slightly below) $\widetilde{p}_{2}^{U}=p_{1}^{U}-\frac{n_{1}^{2}}{2 b}\left(\frac{a-b c}{n_{1}+1}\right)^{2}<0$. Simultaneously, platform provider 2 could set an entry fee $\widetilde{p}_{2}^{D}$ such that $\frac{1}{b}\left(\frac{a-b c}{\widetilde{n}_{2}+1}\right)^{2}-f-\widetilde{p}_{2}^{D}=\max \left(0,-f-p_{1}^{D}\right)$, that is, we must have $\widetilde{p}_{2}^{D}=\frac{1}{b}\left(\frac{a-b c}{\widetilde{n}_{2}+1}\right)^{2}-f$. To solve for the optimal number of developers to be attracted, notice that

$$
\Pi_{2}\left(\widetilde{n}_{2}\right)=\widetilde{p}_{2}^{U}+\left(\frac{1}{b}\left(\frac{a-b c}{\widetilde{n}_{2}+1}\right)^{2}-f-c^{D}\right) \widetilde{n}_{2}
$$

attains its maximum at some $\widetilde{n}_{2} \in(0,1)$, so accounting for the integer constraint yields that either $\widetilde{n}_{2}=1$ or $\widetilde{n}_{2}=0$. This shows that the optimal deviation for platform provider 2 would yield the following profits:

$$
\widetilde{\Pi}_{2}=p_{1}^{U}-\frac{n_{1}^{2}}{2 b}\left(\frac{a-b c}{n_{1}+1}\right)^{2}+\max \left(0, \frac{(a-b c)^{2}}{4 b}-f-c^{D}\right) .
$$

Therefore, in order for $\left(p_{1}^{U}, p_{1}^{D}\right)$ to constitute an equilibrium strategy for platform provider $1, p_{1}^{U}$ and $n_{1}$ should be the solutions to the following programme (as long as profits are positive in equilibrium):

$$
\begin{aligned}
\max _{\widetilde{p}_{1}^{U}, \widetilde{n}_{1}} \Pi_{1}\left(\widetilde{p}_{1}^{U}, \widetilde{n}_{1}\right) & =\max _{\widetilde{p}_{1}^{U}, \widetilde{n}_{1}}\left[\widetilde{p}_{1}^{U}+\left(\frac{1}{b}\left(\frac{a-b c}{\widetilde{n}_{1}+1}\right)^{2}-f-c^{D}\right) \widetilde{n}_{1}\right] \\
\text { s.t. } \widetilde{p}_{1}^{U} & \leq \frac{\widetilde{n}_{1}^{2}}{2 b}\left(\frac{a-b c}{\widetilde{n}_{1}+1}\right)^{2}+\min \left(0, f+c^{D}-\frac{(a-b c)^{2}}{4 b}\right) \\
\widetilde{p}_{1}^{U} & \leq \frac{\widetilde{n}_{1}^{2}}{2 b}\left(\frac{a-b c}{\widetilde{n}_{1}+1}\right)^{2} .
\end{aligned}
$$

The first constraint says that platform provider 1 sets a price for users such that 2 cannot make positive profit, whereas the second one means that application users find it optimal to trade with platform provider 1 at such prices, given that $\widetilde{n}_{1}$ developers are attracted. The first constraint is more stringent than the second one if and only if $\frac{(a-b c)^{2}}{4 b} \geq f+c^{D}$. If this holds, $\frac{(a-b c)^{2}}{4 b}-\left(f+c^{D}\right)$ represents the benefit that accrues to users owing to the existence of an (inactive) platform provider. This is at the expense of the dominant platform provider, which loses some market power owing to the existence of potential competition. 
Because the most interesting cases from a strategic standpoint are those in which the dominant platform provider would be constrained by the existence of its competitor, we assume that $\frac{(a-b c)^{2}}{4 b} \geq f+c^{D}$ and we can ignore the second constraint. Clearly, the first constraint must be satisfied with equality in equilibrium, so platform provider 1 solves:

$$
\max _{\widetilde{n}_{1}} \Pi_{1}\left(\widetilde{n}_{1}\right)=\max _{\widetilde{n}_{1}}\left[\frac{(a-b c)^{2}}{2 b}\left(1-\frac{1}{\left(\widetilde{n}_{1}+1\right)^{2}}\right)-\left(f+c^{D}\right) \widetilde{n}_{1}+f+c^{D}-\frac{(a-b c)^{2}}{4 b}\right] .
$$

Hence, the optimal number of developers pursued by platform provider 1 is $\widetilde{n}_{1}^{*}=\left(\frac{(a-b c)^{2}}{b\left(f+c^{D}\right)}\right)^{\frac{1}{3}}-$ 1 , and $\Pi_{1}\left(\widetilde{n}_{1}^{*}\right) \geq \Pi_{1}(1)>0$.

It can also be shown that users may be subsidized by the dominant platform provider. Some algebra yields that

$$
\widetilde{p}_{1}^{*}=\frac{(a-b c)^{2}}{4 b}-\frac{2(a-b c)\left((a-b c) b\left(f+c^{D}\right)\right)^{\frac{1}{3}}}{2 b}+\frac{\left((a-b c) b\left(f+c^{D}\right)\right)^{\frac{2}{3}}}{2 b}+\left(f+c^{D}\right) .
$$

Taking into account that $\frac{(a-b c)^{2}}{b\left(f+c^{D}\right)} \geq 4$, it can be shown that buyers are subsidized if and only if it holds that $8 \leq \frac{(a-b c)^{2}}{b\left(f+c_{D}\right)} \leq \frac{4}{3 \sqrt{3}-5}$. For low values of $(a-b c)^{2} / b\left(f+c^{D}\right)$, it is pointless for the dominant platform provider to subsidize users since the low surplus that could be extracted by the competing platform provider via developers would not compensate the losses it would make when attracting users. This changes as the surplus grows, so the dominant platform provider prices the competitor out by subsidizing users. However, for large enough surplus, users are not subsidized.

\subsubsection{Profit comparison}

Because the unique equilibrium when platforms are compatible is the symmetric one derived in Section 3 and profits in that equilibrium vanish as $t \downarrow 0$, we have proved the following:

Proposition 5 Suppose there exist two competing platform providers that sell incompatible platforms. If $t=0$, there exist (two) asymmetric equilibria in which one of the platform providers captures all users and achieves a positive profit that exceeds that achieved when platforms are compatible. Moreover, users are subsidized by the dominant platform provider if and only if $8 \leq \frac{(a-b c)^{2}}{b\left(f+c_{D}\right)} \leq \frac{4}{3 \sqrt{3}-5}$. 
The striking result that platform compatibility yields greater profits than incompatibility in a symmetric equilibrium is highly dependent on the type of equilibrium played. Indeed, asymmetric equilibria when platforms are incompatible may yield greater profits for the dominant platform provider, as we just showed.

\subsubsection{Social efficiency}

We now compare social efficiency between competing platforms when they are compatible and when they are incompatible and play the asymmetric equilibrium derived in Section 5.1.2. We begin with a simple remark.

Remark 2 Assume that $t=0$ and suppose there exist two competing platform providers. (a) If platforms are compatible, then total welfare in the unique equilibrium is

$$
W^{c o m}\left(N^{*}\right)=\frac{(a-b c)^{2}}{2 b}\left(1-\frac{1}{\left(N^{*}+1\right)^{2}}\right)-\left(f+c^{D}\right) N^{*} .
$$

(b) If platforms are incompatible, then total welfare in the cornering equilibrium derived in Section 5.1.2 is

$$
\tilde{W}^{i n c}\left(\widetilde{n}_{1}^{*}\right)=\frac{(a-b c)^{2}}{2 b}\left(1-\frac{1}{\left(\widetilde{n}_{1}^{*}+1\right)^{2}}\right)-\left(f+c^{D}\right) \widetilde{n}_{1}^{*}
$$

$N^{*}$ and $\widetilde{n}_{1}^{*}$ are the equilibrium number of developers in each case.

Proof. See Appendix.

Now, because $\widetilde{n}_{1}^{*}=\left(\frac{(a-b c)^{2}}{b\left(f+c^{D}\right)}\right)^{\frac{1}{3}}-1\left(\right.$ see Section 5.1.2 and $N^{*}=\left(\frac{(a-b c)^{2}}{b\left(f+c^{D}\right)}\right)^{\frac{1}{2}}-1$ (see Section 3), simple algebra shows that $\tilde{W}^{i n c}\left(\widetilde{n}_{1}^{*}\right)>W^{\text {com }}\left(N^{*}\right)$. Therefore, when $t=0$ the asymmetric equilibrium under incompatibility generates larger welfare than the unique equilibrium under compatibility.

The advantage of compatibility versus asymmetric incompatibility is that it addresses better the idiosyncratic tastes of users (horizontal dimension) because both platforms are active and each serves half of the market. In the asymmetric equilibrium, only one platform is active and those users located far away derive limited utility from applications on that platform. We will show that the advantage of compatibility lessens as $t$ declines. The 
disadvantage of compatibility is that it induces too much entry by developers. The excessive entry is independent of horizontal differentiation because $p^{D *}=c^{D}$ regardless of the value of $t$. Therefore, when $t=0$, asymmetric incompatibility has no disadvantage compared to compatibility and thus the social welfare that it generates is larger.

\subsection{Heterogeneous users $(t>0)$}

\subsubsection{Asymmetric equilibria when platforms are compatible}

As argued in Section 5.1.1, when studying asymmetric equilibria with compatibility, we may (without loss of generality) consider the case where platform 1 charges $p_{1}^{D}<c^{D}$ and $p_{1}^{D}<p_{2}^{D}$. Moreover, a necessary condition for an asymmetric equilibrium to exist is that the system of five equations formed by (17)-(21) and five unknowns $\left(p_{1}^{U}, p_{1}^{D}, p_{2}^{U}, x\right.$, and $\left.N\right)$ be satisfied.

Given the difficulty of solving that system algebraically, we have performed a large volume of numeric simulations. ${ }^{23}$ The simulations all suggest that there is no asymmetric equilibrium in the case of compatible platforms (if one requires that $N$ be larger than or equal to 1 when solving the system of equations). Intuitively, because compatibility prevents platforms from differentiating based on the number of applications that run on them, given $N$ and the assumption that of symmetric horizontal differentiation between platforms, the prices charged to users have a strong propensity to equalization. But when $p_{1}^{U}=p_{2}^{U}$, platform 1 prefers to increase $p_{1}^{D}$ to stop hemorrhaging losses on the developer side that are not compensated by additional gains on the user side.

\subsubsection{Asymmetric equilibria when platforms are incompatible}

We now generalize the development in Section 5.1 .2 to the case $t>0$.

Suppose that $\left\{\left(p_{i}^{U}, p_{i}^{D}\right)\right\}_{i=1}^{2}$ is such that platform 1 completely dominates the market. What is the most profitable deviation that platform 2 could undertake? To capture $x_{2}$ users, it should charge a price $p_{2}^{U}$ such that $\frac{\left(1-t\left(1-x_{2}\right)\right) n_{1}^{2}}{2 b}\left(\frac{a-b c}{n_{1}+1}\right)^{2}-p_{1}^{U}<-p_{2}^{U}$, that is, $\widetilde{p}_{2}^{U}=p_{1}^{U}-\frac{\left(1-t\left(1-x_{2}\right)\right) n_{1}^{2}}{2 b}\left(\frac{a-b c}{n_{1}+1}\right)^{2}$. Using the free-entry condition for developers yields that platform 2's most profitable deviation is given by the solution to the following

\footnotetext{
${ }^{23}$ The Mathematica code is available from the authors.
} 
programme:

$\max _{x_{2}, n_{2}} x_{2}\left(p_{1}^{U}-\frac{\left(1-t\left(1-x_{2}\right)\right)(a-b c)^{2}}{2 b}\left(\frac{n_{1}}{n_{1}+1}\right)^{2}\right)+n_{2}\left(\frac{x_{2}\left(2-t x_{2}\right)(a-b c)^{2}}{2 b}\left(\frac{1}{n_{2}+1}\right)^{2}-\left(f+c^{D}\right)\right)$.

The first-order conditions are

$$
\begin{aligned}
\frac{\left(1-t x_{2}\right)(a-b c)^{2} n_{2}}{b}\left(\frac{1}{n_{2}+1}\right)^{2}+p_{1}^{U}-\frac{\left(1-t+2 t x_{2}\right)(a-b c)^{2}}{2 b}\left(\frac{n_{1}}{n_{1}+1}\right)^{2} & =0 \\
\frac{x_{2}\left(2-t x_{2}\right)(a-b c)^{2}\left(1-n_{2}\right)}{2 b\left(n_{2}+1\right)^{3}}-\left(f+c^{D}\right) n_{2} & =0 .
\end{aligned}
$$

The second first-order condition, together with the integer constraint, implies that platform 2 finds it optimal to promote either no entry or entry by just one developer. If it finds it optimal to promote entry by one developer, then it chooses to serve $x_{2}=$

$$
\begin{aligned}
& \frac{1-2(1-t)\left(\frac{n_{1}}{n_{1}+1}\right)^{2}+\frac{4 b p_{1}^{U}}{(a-b c)^{2}}}{t\left(1+4\left(\frac{n_{1}}{n_{1}+1}\right)^{2}\right)} \text { users. Note that } x_{2}>0 \text { if and only if } \\
& p_{1}^{U}>\frac{(a-b c)^{2}\left(2(1-t)\left(\frac{n_{1}}{n_{1}+1}\right)^{2}-1\right)}{4 b}=\frac{(a-b c)^{2}\left(2(1-t)\left(1-\sqrt{\frac{2 b\left(p_{1}^{D}+f\right)}{(2-t)(a-b c)^{2}}}\right)^{2}-1\right)}{4 b} .
\end{aligned}
$$

If $x_{2}>0$, then profit is

$$
x_{2}\left(p_{1}^{U}-\frac{\left(1-t\left(1-x_{2}\right)\right)(a-b c)^{2}}{2 b}\left(\frac{n_{1}}{n_{1}+1}\right)^{2}\right)+\frac{x_{2}\left(2-t x_{2}\right)(a-b c)^{2}}{8 b}-\left(f+c^{D}\right)
$$

Therefore, the equilibrium $p_{1}^{U}$ and $p_{1}^{D}$ solve the following constrained optimization program:

$$
\begin{aligned}
& \max _{p_{1}^{U}, p_{1}^{D}} p_{1}^{U}+\left(p_{1}^{D}-c^{D}\right)\left(\sqrt{\frac{(2-t)(a-b c)^{2}}{2 b\left(p_{1}^{D}+f\right)}}-1\right) \\
& \text { s.t. } \quad x_{2}\left(p_{1}^{U}-\frac{\left(1-t\left(1-x_{2}\right)\right)(a-b c)^{2}}{2 b}\left(1-\sqrt{\frac{2 b\left(p_{1}^{D}+f\right)}{(2-t)(a-b c)^{2}}}\right)^{2}\right)+\frac{x_{2}\left(2-t x_{2}\right)(a-b c)^{2}}{8 b}-\left(f+c^{D}\right) \leq 0 \\
& p_{1}^{U} \leq \frac{(1-t)(a-b c)^{2}}{2 b}\left(\frac{n_{1}}{n_{1}+1}\right)^{2}
\end{aligned}
$$




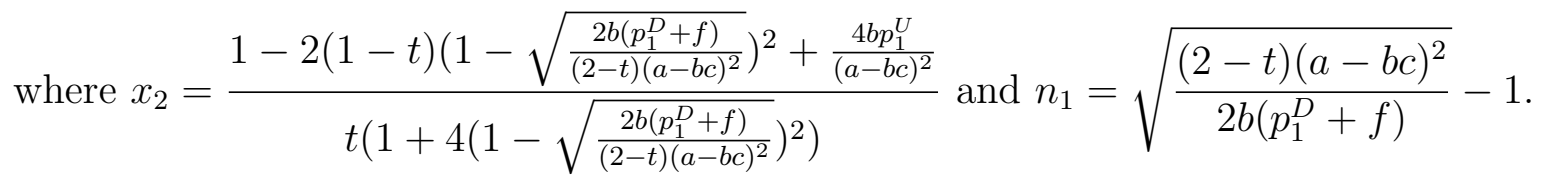

The first constraint prevents platform 2 from deviating from a situation where it obtains no demand. The second constraint guarantees that all users purchase platform 1.

\subsubsection{Profit comparison}

While the constrained optimization problem that we have just set up is hard to solve in general, numerical analysis is manageable ${ }^{24}$ The simulations (Figure 3 ) show that when $t$ is low, the dominant platform earns larger profits than symmetric platforms under compatibility. When $t$ is low, the equilibrium under compatibility displays very intense competition for users (in addition to the Bertrand-type competition for developers discussed in Section 3), as the platforms are symmetric and scarcely differentiated. In the asymmetric incompatible case, the winning platform needs to lower prices to attract buyers that are "far away" but, since $t$ is low, the lack of platform appeal for which they need to be compensated is also low. The optimistic expectations towards the winning platform allow it to vertically differentiate its offering based on the number of applications that it offers. The vertical differentiation, in turn, allows for relatively large access prices and high profit.

As $t$ grows and horizontal differentiation intensifies, it becomes harder for the dominant platform to attract all users. This forces the dominant platform to lower $p^{U}$ and profits decline. In fact, Figure 3 shows that when $t$ is large, dominant platform equilibria may generate lower profits than the symmetric equilibrium under incompatibility.

We also note that if side payments were feasible, one necessary condition for platforms to agree on compatibility through bargaining would be that industry profits under compatibility exceeded the profits of the dominant platform under incompatibility. Figure 3 shows that when $t$ is low, dominant platform profits are more than twice the profits under compatibility. Therefore, when $t$ is low it is impossible for platforms to agree on compatibility.

Figure 3 is also useful in extending the conclusions of our model to those situations in which compatibility softens the horizontal differentiation of competing platforms because of standardization. Thus, if platforms are allowed to switch from compatibility to incompatibility (or vice versa), there are typically two distinct effects on the nature of platform competition. First, the size of the relevant network changes and, thus, the equilibrium prices

\footnotetext{
${ }^{24}$ The Mathematica code is available from the authors. The figure shows profits under compatibility, symmetric incompatibility, and asymmetric incompatibility at different values of $t \in[0,2 / 3)$.
} 


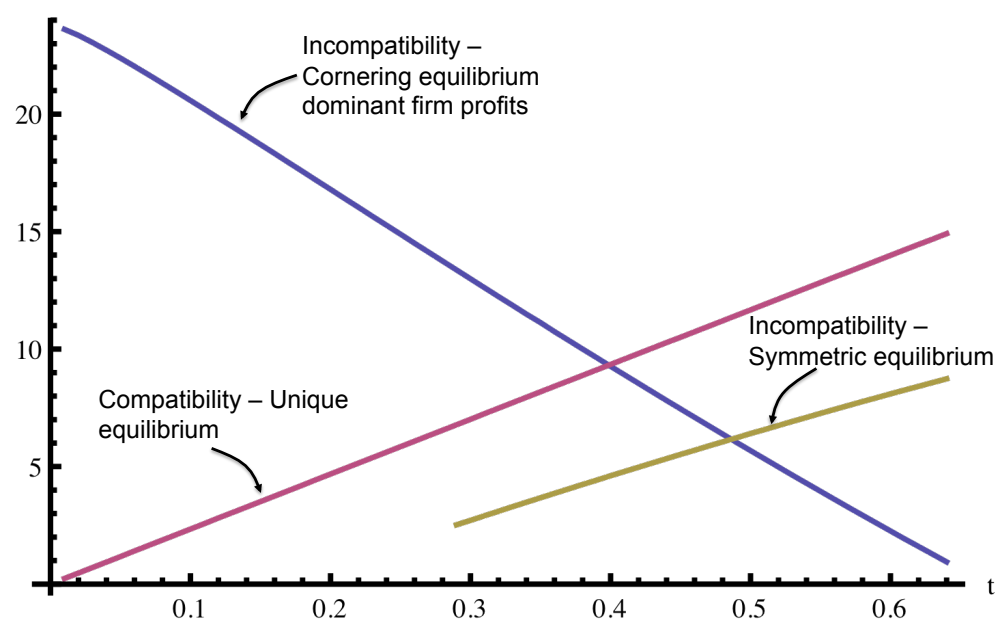

Figure 3: Profit comparison. Parameters: $\frac{(a-b c)^{2}}{2 b}=50, c^{D}=0.1$, and $f=0$.

also change (this has been the issue that we have investigated in this paper). Second, $t$ may also change in value in the case of platform standardization. In particular, $t$ is likely to be lower when platforms are compatible. By fixing the value of $t$ in our analysis, we have been able to isolate the first effect to better understand it.

While future research may carefully address the issue of changing $t$, based on Figure 3 we can safely conclude that small changes in $t$ have no qualitative effects on our results. To see this, note that a decrease in $t$ for the compatible case is equivalent to a rotation downwards of the compatible profit line from the origin. If there were large changes in $t$, however, then profits under symmetric incompatibility may be larger than under compatibility, reversing one of our main results.

\subsubsection{Social efficiency}

We now compare social efficiency between competing platforms when they are compatible, when they are incompatible and play the unique symmetric equilibrium derived in Section 4 , and when they are incompatible and play the asymmetric equilibrium derived in Section 5.2.2. The following is a generalization of Remark 2 to the cases with $t>0$.

Remark 3 Let $A=\frac{(a-b c)^{2}}{2 b}$. Suppose there exist two competing platform providers. (a) If platforms are compatible, then total welfare in the unique equilibrium is

$$
W^{c o m}\left(N^{*}\right)=\left(1-\frac{t}{4}\right) A\left(1-\frac{1}{\left(N^{*}+1\right)^{2}}\right)-\left(f+c^{D}\right) N^{*}
$$


(b) If platforms are incompatible, then total welfare in the unique symmetric equilibrium is

$$
W^{i n c}\left(n^{* *}\right)=\left(1-\frac{t}{4}\right) A\left(1-\frac{1}{\left(n^{* *}+1\right)^{2}}\right)-2\left(f+c^{D}\right) n^{* *}
$$

(c) If platforms are incompatible, then total welfare in the cornering equilibrium derived in Section 5.2.2 is

$$
\tilde{W}^{i n c}\left(\widetilde{n}_{1}^{*}\right)=\left(1-\frac{t}{2}\right) A\left(1-\frac{1}{\left(\widetilde{n}_{1}^{*}+1\right)^{2}}\right)-\left(f+c^{D}\right) \widetilde{n}_{1}^{*} .
$$

$N^{*}, n^{* *}$, and $\widetilde{n}_{1}^{*}$ are the equilibrium number of developers in each case.

Proof. See Appendix.

Because both $n^{* *}$ and $\widetilde{n}_{1}^{*}$ depend on $p^{D * *}$ and $\widetilde{p}_{1}^{D}$ for which we do not have explicit solutions, we proceed to compare $W^{c o m}\left(N^{*}\right), W^{i n c}\left(n^{* *}\right)$, and $\tilde{W}^{i n c}\left(\widetilde{n}_{1}^{*}\right)$ through numerical simulations ${ }^{25}$ The examples reveal that the comparison is ambiguous (see Figures 4 and 5 below). When $t$ is low, there is no symmetric incompatible equilibrium. In this case, the asymmetric incompatible equilibrium appears to generate larger surplus than compatibility. The intuition is exactly as presented in Section 5.1.4.

We also see that symmetric incompatibility may produce larger social surplus than compatibility. While compatibility saves on costs to build a given network of developers, it leads to excessive entry.

Finally, the simulations show that welfare is a decreasing function of $t$, regardless of the intellectual property regime and equilibrium considered. As the intensity of preferences grows, users experience increased disutility from purchasing a platform that is not exactly of their liking.

\section{Conclusion}

We have studied price competition between providers of two-sided platforms in a setting where the two sides first pay a price to gain access to the functionalities of a platform and

\footnotetext{
${ }^{25}$ The Mathematica code is available from the authors.
} 


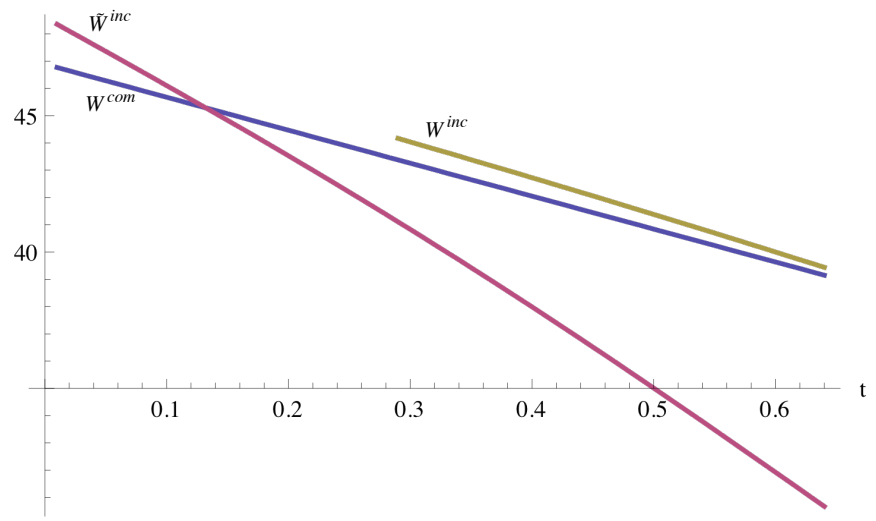

Figure 4: Total welfare comparison. Parameters: $\frac{(a-b c)^{2}}{2 b}=50, c^{D}=0.1$, and $f=0$.

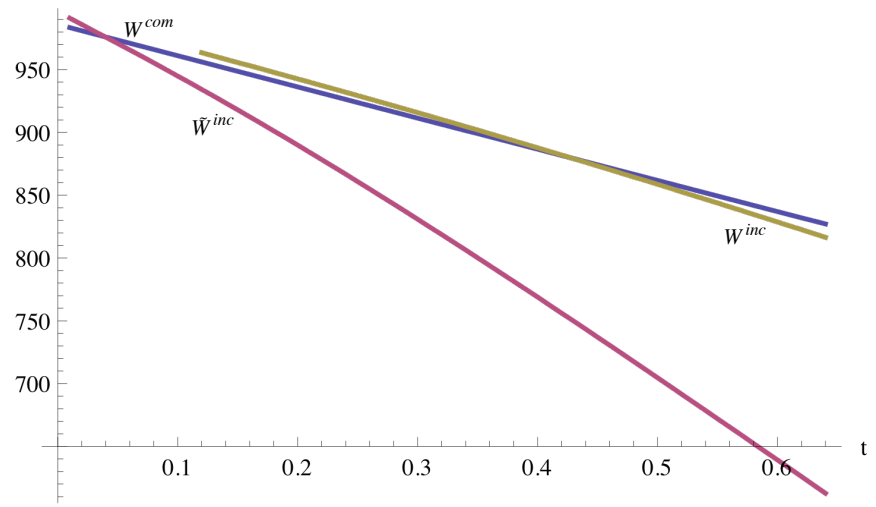

Figure 5: Total welfare comparison. Parameters: $\frac{(a-b c)^{2}}{2 b}=1000, c^{D}=0.1$, and $f=0$.

then interact with each other under oligopolistic conditions. The paper provides a theory for why firms may choose to make their platforms incompatible, despite the softer price competition for users that ensues under compatibility. Incompatibility might lead to market dominance and high profits by one of the platform providers, even if they are both ex ante identical and there are not fixed costs of operation. We have also shown that symmetric and asymmetric equilibria under incompatibility may exhibit user subsidization in order to spur developer entry, a result that seems in line with analysts' observations in many markets with two-sided platforms. Finally, we have shown that platform competition results in socially insufficient (excessive) developer entry under incompatibility (compatibility).

One limitation of our approach is that we did not allow platform providers to charge royalty fees per unit of output sold to developers (in addition to the fixed access fee $p_{i}^{D}$ ). There is a strong incentive in our model to set such royalties below zero. On the one hand, incompatible platforms partly internalize the effects of imperfect competition downstream. 
Therefore, they will want to distort second-period trade as little as possible. Negative royalty fees would move such trade closer to efficiency. Compatible platforms, on the other hand, are likely to set royalty fees below zero for strategic rather than efficiency reasons. ${ }^{26}$ Negative royalties would allow developers which traded with a given platform provider to become tougher competitors in the second period, which would boost profits on the developer side because trade by developers with the competing platform provider would be discouraged. This effect would be reversed if second-period competition displayed strategic complementarity.

A second shortcoming of our approach is the assumption that products sold by developers are homogeneous. Allowing for product differentiation would introduce an additional incentive for the platform provider/s to promote developer entry. By doing so, developers could better address users' tastes, and hence the platform provider/s could extract the increase in developer profits and user utility by raising license/entry fees and the price charged to users for the platform. However, the main insights regarding the relative advantages of compatibility vs. incompatibility and social efficiency are likely to be qualitatively unaffected.

Perhaps a more important limitation is our focus on the cases in which compatibility requires all platform providers to agree. This may be representative for technological standards, but in several contexts, a platform can be made compatible with another one by means of an adapter. It would be interesting to examine the situations in which the developers for one of the platforms can sell applications to users of both platforms, but the developers for the competing platform can sell applications only to users of their platform. Although technically challenging, analyzing the private and social incentives for partial compatibility seems an important avenue for future research on compatibility in markets with two-sided platforms.

\footnotetext{
${ }^{26}$ We are implicitly assuming that both types of agents trade with a platform provider simultaneously so as to focus on the strategic or efficiency reasons why platform providers may want to keep royalties low. We are cognizant that this sidesteps the remarkable result by Hagiu (2006a) that platform providers may wish to use royalty fees to alleviate a hold-up problem when developers decide whether or not to trade with a platform provider before users do.
} 


\section{Appendix}

Proof of Proposition 3. Letting

$$
\begin{aligned}
H(x) & \equiv \frac{2(1-t x)^{2}\left(a-b c-\sqrt{\frac{2 b\left(p_{1}^{D}+f\right)}{x(2-t x)}}\right) \sqrt{\frac{2 b\left(p_{1}^{D}+f\right)}{[x(2-t x)]^{3}}}-t\left(a-b c-\sqrt{\frac{2 b\left(p_{1}^{D}+f\right)}{x(2-t x)}}\right)^{2}}{2 b}+ \\
& \frac{2(1+t x-t)^{2}\left(a-b c-\sqrt{\frac{2 b\left(p_{2}^{D}+f\right)}{(1-x)(2-t(1-x))}}\right) \sqrt{\frac{2 b\left(p_{2}^{D}+f\right)}{[(1-x)(2-t(1-x))]^{3}}}-t\left(a-b c-\sqrt{\frac{2 b\left(p_{2}^{D}+f\right)}{(1-x)(2-t(1-x))}}\right)^{2}}{2 b},
\end{aligned}
$$

we have that the implicit function theorem applied on (14) yields

$$
\frac{\partial x\left(p_{1}^{U}, p_{1}^{D}\right)}{\partial p_{1}^{U}}=\frac{1}{H(x)}
$$

and

$$
\frac{\partial x\left(p_{1}^{U}, p_{1}^{D}\right)}{\partial p_{1}^{D}}=\frac{\frac{(1-t x)(a-b c)}{\sqrt{2 b x(2-t x)\left(p_{1}^{D}+f\right)}}-\frac{(1-t x)}{x(2-t x)}}{H(x)}
$$

Given that we restrict our attention to symmetric equilibria in which $x=\frac{1}{2}, p_{1}^{U}=p_{2}^{U} \equiv p^{U}$ and $p_{1}^{D}=p_{2}^{D} \equiv p^{D}$, the first-order conditions (15) and (16) can be simplified with the aid of expressions (22) and (23) so as to get:

$$
\frac{1}{2}=\frac{2 b\left(p^{U}+\frac{\left(p^{D}-c^{D}\right)(a-b c)(2-t)}{\sqrt{2 b\left(p^{D}+f\right)(4-t)}}\right)}{2 t\left(a-b c-\sqrt{\frac{8 b\left(p^{D}+f\right)}{4-t}}\right)^{2}-\frac{4(2-t)^{2}}{4-t}\left(a-b c-\sqrt{\frac{8 b\left(p^{D}+f\right)}{4-t}}\right) \sqrt{\frac{8 b\left(p^{D}+f\right)}{4-t}}}
$$

and

$$
\begin{aligned}
& \frac{2 b\left(p^{U}+\frac{\left(p^{D}-c^{D}\right)(a-b c)(2-t)}{\sqrt{2 b\left(p^{D}+f\right)(4-t)}}\right)\left(\frac{(2-t)(a-b c)}{\sqrt{2 b(4-t)\left(p^{D}+f\right)}}-\frac{2(2-t)}{4-t}\right)}{\frac{4(2-t)^{2}}{4-t}\left(a-b c-\sqrt{\frac{8 b\left(p^{D}+f\right)}{(4-t)}}\right) \sqrt{\frac{8 b\left(p^{D}+f\right)}{4-t}}-2 t\left(a-b c-\sqrt{\frac{8 b\left(p^{D}+f\right)}{4-t}}\right)^{2}}-\frac{\left(p^{D}-c^{D}\right)(a-b c) \sqrt{4-t}}{4 \sqrt{2 b\left(p^{D}+f\right)^{3}}}= \\
& 1-\frac{(a-b c) \sqrt{4-t}}{2 \sqrt{2 b\left(p^{D}+f\right)}}
\end{aligned}
$$


Plugging equation (24) into 25 yields

$$
\frac{(a-b c) \sqrt{4-t}}{8}\left(\frac{4\left(f+c^{D}\right)+t\left(p^{D}-c^{D}\right)}{\sqrt{2 b\left(p^{D}+f\right)^{3}}}\right)=1
$$

whence it is relatively simple to show that there exists a unique value of $p^{D}$ that solves such equation 27 Denoting such a value of $p^{D}$ by $p^{D * *}$, note that $p^{D * *}>c^{D}$ if and only if $\frac{(a-b c)^{2}}{2 b}>\frac{4\left(f+c^{D}\right)}{4-t}$ holds.

Using (24) and (26), we can find the price $p^{U * *}$ charged to users in equilibrium by performing some algebra:

$p^{U * *}=\frac{4 t\left(4+2 t-t^{2}\right)\left(p^{D * *}+f\right)\left(p^{D * *}-c^{D}\right)^{2}-32(2-t)(3-t)\left(f+c^{D}\right)\left(p^{D * *}+f\right)\left(p^{D * *}-c^{D}\right)}{(4-t)\left[4\left(f+c^{D}\right)+t\left(p^{D * *}-c^{D}\right)\right]^{2}}$.

In order for both platform providers charging a pair of prices $\left(p^{U * *}, p^{D * *}\right)$ to constitute an equilibrium, the values of the parameter space should be such that some conditions hold. Thus, the marginal user (i.e., the one located at the middle of the segment) should attain a positive utility, platform providers should make positive profits and a unilateral increase in the user access fee should not increase user demand for the platform that raises such price (as required by the monotonicity refinement). We proceed to derive the parameter restrictions implied by each of these equilibrium conditions.

First, observe that the marginal user attains a positive utility if and only if $u_{\hat{j}}^{\text {inc }}(1 / 2)>$ $p^{U * *}$. Because $u_{\hat{j}}^{i n c}(1 / 2)=\frac{(2-t)\left(a-b c-\sqrt{\left.\frac{8 b\left(p^{D * *}+f\right)}{4-t}\right)^{2}}\right.}{4 b}$, expression 266 implies that this condition is equivalent to the following one:

$$
\frac{2(2-t)(4-t)\left(p^{D * *}-c^{D}\right)^{2}\left(p^{D * *}+f\right)}{\left[4\left(f+c^{D}\right)+t\left(p^{D * *}-c^{D}\right)\right]^{2}}>p^{U * *}
$$

Making use of (27), we have that this inequality is satisfied if and only if the following holds:

$$
\left(t^{3}+6 t^{2}-40 t+32\right)\left(p^{D * *}-c^{D}\right)^{2}+16(2-t)(3-t)\left(f+c^{D}\right)\left(p^{D * *}-c^{D}\right)>0 .
$$

\footnotetext{
${ }^{27}$ Letting $g\left(p^{D}\right) \equiv \frac{(a-b c) \sqrt{4-t}}{8}\left(\frac{4\left(f+c^{D}\right)+t\left(p^{D}-c^{D}\right)}{\sqrt{2 b\left(p^{D}+f\right)^{3}}}\right)$ in expression $\sqrt{26}$, the result follows because $g(-f)=\infty, g(\infty)=0$ and $g^{\prime}<0\left(\right.$ since $\left.t<\frac{2}{3}<4\right)$.
} 
The left hand side is strictly convex in $p^{D * *}$ (since $t<2 / 3$ ) and takes a negative value for $p^{D * *}=-f$ (which clearly is the smallest admissible value of $p^{D * *}$ ), so it follows that the marginal user makes a positive utility in equilibrium if and only if $p^{D * *}>c^{D}$, that is, if and only if $\frac{(a-b c)^{2}}{2 b}>\frac{4\left(f+c^{D}\right)}{4-t}$ is satisfied. Hence, the parameter constraint that users attain a positive utility implies that developers are not subsidized to enter the market in a symmetric equilibrium with incompatible platforms.

Second, we study the conditions under which platform providers make non-negative profits in a symmetric equilibrium. Profits made by each of them are equal to

$$
\Pi_{1}^{* *}=\Pi_{2}^{* *} \equiv \Pi^{* *}=\frac{p^{U * *}}{2}+\left(p^{D * *}-c^{D}\right)\left(\frac{(a-b c) \sqrt{4-t}}{2 \sqrt{2 b\left(p^{D * *}+f\right)}}-1\right) .
$$

By (27) and (26), we have

$$
\begin{aligned}
\Pi^{* *}= & \frac{\left(p^{D * *}-c^{D}\right)}{(4-t)\left[4\left(f+c^{D}\right)+t\left(p^{D * *}-c^{D}\right)\right]^{2}}\left[2 t\left(4+2 t-t^{2}\right)\left(p^{D * *}+f\right)\left(p^{D * *}-c^{D}\right)-\right. \\
& \left.16(2-t)(3-t)\left(f+c^{D}\right)\left(p^{D * *}+f\right)+4(4-t)^{2}\left(f+c^{D}\right)\left(p^{D * *}-c^{D}\right)+t(4-t)^{2}\left(p^{D * *}-c^{D}\right)^{2}\right],
\end{aligned}
$$

so note that $\Pi^{* *} \geq 0$ if and only if $h\left(p^{D * *}\right) \geq 0$, where

$$
\begin{gathered}
h\left(p^{D * *}\right) \equiv t\left(24-4 t-t^{2}\right)\left(p^{D * *}-c^{D}\right)^{2}+2\left(4 t^{2}-t^{3}+32-12 t\right)\left(p^{D * *}-c^{D}\right)\left(f+c^{D}\right)- \\
16(2-t)(3-t)\left(p^{D * *}+f\right)\left(f+c^{D}\right) .
\end{gathered}
$$

Because $h^{\prime \prime}>0$ and $h(-f)<0$, it follows that $h\left(p^{D * *}\right) \geq 0$ for sufficiently large $p^{D * *}$, or equivalently, for large enough $\frac{(a-b c)^{2}}{2 b}$. Let us refer to such a value of $\frac{(a-b c)^{2}}{2 b}$ as $W, 28$ Because $h\left(c^{D}\right)<0$, the condition that equilibrium profit is positive is more stringent than

\footnotetext{
${ }^{28}$ It is straightforward to show that the smallest value of $p^{D * *}$ that makes profits non-negative is $c^{D}+$ $w\left(f+c^{D}\right)$, where$$
w=\frac{2\left(t^{3}+4 t^{2}-28 t+16\right)+\sqrt{\left(t^{3}+4 t^{2}-28 t+16\right)^{2}+64 t(2-t)(3-t)\left(24-4 t-t^{2}\right)}}{2 t\left(24-4 t-t^{2}\right)} .
$$

The lower bound on $\frac{(a-b c)^{2}}{2 b}$ can be obtained by using this value for $p^{D}$ in equation 26 , whence we have that the following condition should hold:

$$
\frac{(a-b c)^{2}}{2 b} \geq \frac{64(1+w)^{3}\left(f+c^{D}\right)}{(4-t)(4+t w)^{2}} \equiv W
$$


the one that makes the utility of the marginal user positive (which simply requires that $\left.p^{D * *}>c^{D}\right)$, so $W>\frac{4\left(f+c^{D}\right)}{4-t}$.

The final condition that must hold in a symmetric equilibrium is the monotonicity condition, which requires that $\partial x\left(p_{1}^{U}, p_{1}^{D}\right) / \partial p_{1}^{U}<0$ and $\partial x\left(p_{1}^{U}, p_{1}^{D}\right) / \partial p_{1}^{D}<0$ both hold at the equilibrium prices. It is easy to prove that both $\partial x\left(p_{1}^{U}, p_{1}^{D}\right) / \partial p_{1}^{U}$ and $\partial x\left(p_{1}^{U}, p_{1}^{D}\right) / \partial p_{1}^{D}$ always have the same sign at the symmetric equilibrium prices. Also, equation (15) implies that $\partial x\left(p_{1}^{U}, p_{1}^{D}\right) / \partial p_{1}^{U}<0$ (evaluated at the symmetric equilibrium prices) if and only if

$$
p_{1}^{U}+\frac{\left(p_{1}^{D}-c^{D}\right)(a-b c)\left(1-t x\left(p_{1}^{U}, p_{1}^{D}\right)\right)}{\sqrt{2 b\left(p_{1}^{D}+f\right) x\left(p_{1}^{U}, p_{1}^{D}\right)\left(2-t x\left(p_{1}^{U}, p_{1}^{D}\right)\right)}}>0 .
$$

Using expressions (26) and (27) implies that the condition in (28) is equivalent to the following one:

$$
\frac{4 t\left(4-t^{2}+4\right)\left(p^{D * *}+f\right)\left(p^{D * *}-c^{D}\right)^{2}-32(2-t)^{2}\left(f+c^{D}\right)\left(p^{D * *}-c^{D}\right)\left(p^{D * *}+f\right)}{(4-t)\left[4\left(f+c^{D}\right)+t\left(p^{D * *}-c^{D}\right)\right]^{2}}>0 .
$$

Therefore, the monotonicity requirement boils down to assuming that $p^{D * *}>c^{D}+\frac{8(2-t)^{2}\left(f+c^{D}\right)}{t\left(8-t^{2}\right)}$, which can be shown to directly satisfy the non-negativity constraint on equilibrium profits for $t<2 / 3\left(\right.$ since $\left.h\left(c^{D}+\frac{8(2-t)^{2}\left(f+c^{D}\right)}{t\left(8-t^{2}\right)}\right)>0\right)$. Note by 26 that $p^{D * *} \geq c^{D}+$ $\frac{8(2-t)^{2}\left(f+c^{D}\right)}{t\left(8-t^{2}\right)}$ if and only if $\frac{(a-b c)^{2}}{2 b} \geq Z^{\prime} \equiv \frac{4\left(f+c^{D}\right)\left(32+8 t^{2}-24 t-t^{3}\right)^{3}}{t^{3}\left(8-t^{2}\right)(4-t)^{5}}$.

To conclude the proof, we examine the conditions under which users of applications may be subsidized when trading with a platform provider. Observe that expression (27) and the fact that $p^{D * *}>c^{D}$ together imply that $p^{U * *} \leq 0$ if and only if

$$
t\left(4+2 t-t^{2}\right)\left(p^{D * *}-c^{D}\right)-8(2-t)(3-t)\left(f+c^{D}\right) \leq 0
$$

that is, users are subsidized in equilibrium if and only if the following inequality holds:

$$
p^{D * *} \leq c^{D}+\frac{8(2-t)(3-t)\left(f+c^{D}\right)}{t\left(4+2 t-t^{2}\right)} .
$$


Letting $p^{D}=c^{D}+\frac{8(2-t)(3-t)\left(f+c^{D}\right)}{t\left(4+2 t-t^{2}\right)}$ in the left hand side of expression 26 yields

$$
\frac{(a-b c) \sqrt{4-t}}{8}\left(\frac{4\left(f+c^{D}\right)+\frac{8(2-t)(3-t)\left(f+c^{D}\right)}{\left(4+2 t-t^{2}\right)}}{\sqrt{2 b\left(f+c^{D}+\frac{8(2-t)(3-t)\left(f+c^{D}\right)}{t\left(4+2 t-t^{2}\right)}\right)^{3}}}\right),
$$

which is not larger than 1 for $\frac{(a-b c)^{2}}{2 b} \leq \frac{4\left(f+c^{D}\right)\left(t^{2}-6 t+12\right)^{3}}{(4-t)^{2} t^{3}\left(4+2 t-t^{2}\right)} \equiv Z$. This shows that $p^{U * *} \leq 0$ if and only if $\frac{(a-b c)^{2}}{2 b} \leq Z$. Because $Z>Z^{\prime}$ for $t<2 / 3$, we have that users are subsidized if $\frac{(a-b c)^{2}}{2 b} \in\left[Z^{\prime}, Z\right)$, whereas they are not subsidized if $\frac{(a-b c)^{2}}{2 b} \geq Z$.

Proof of Remark 1. We would like to see how many solutions $\left(x, n_{1}, n_{2}\right)$ the following system has:

$$
\begin{gathered}
\frac{(1-t x) n_{1}^{2}}{2 b}\left(\frac{a-b c}{n_{1}+1}\right)^{2}-p^{U}=\frac{(1-t(1-x)) n_{2}^{2}}{2 b}\left(\frac{a-b c}{n_{2}+1}\right)^{2}-p^{U}, \\
p^{D}=\frac{x(2-t x)}{2 b}\left(\frac{a-b c}{n_{1}+1}\right)^{2}-f \\
p^{D}=\frac{(1-x)(2-t(1-x))}{2 b}\left(\frac{a-b c}{n_{2}+1}\right)^{2}-f .
\end{gathered}
$$

Note that we are imposing symmetry $\left(p_{1}^{U}=p_{2}^{U}=p^{U}\right.$ and $\left.p_{1}^{D}=p_{2}^{D}=p^{D}\right)$ because we study symmetric equilibria only.

Notice first that

$$
x=\frac{1}{2} \quad \text { and } \quad n_{1}=n_{2}=\frac{\sqrt{b(a-b c)^{2}\left(p^{D}+f\right)\left(2-\frac{t}{2}\right)}}{2 b\left(p^{D}+f\right)}-1
$$

is one solution. The question is whether there are asymmetric solutions (solutions with $x \neq \frac{1}{2}$ and $\left.n_{1} \neq n_{2}\right)$.

We begin by working with equation 30 to obtain:

$$
\frac{\left(p^{D}+f\right) 2 b}{x(2-t x)}=\left(\frac{a-b c}{n_{1}+1}\right)^{2} .
$$


Likewise, equation (31) yields:

$$
\frac{\left(p^{D}+f\right) 2 b}{(1-x)(2-t(1-x))}=\left(\frac{a-b c}{n_{2}+1}\right)^{2} \text {. }
$$

Using these two expressions in (29), we have:

$$
\frac{n_{1}}{n_{2}}=\left(\frac{(1-t(1-x)) x(2-t x)}{(1-x)(2-t(1-x))(1-t x)}\right)^{\frac{1}{2}} .
$$

Now, solving for $n_{1}$ in $30 p$, and letting $G \equiv \sqrt{\frac{2 b\left(p^{D}+f\right)}{(a-b c)^{2}}}$, we obtain

$$
n_{1}=\frac{\sqrt{x(2-t x)}}{G}-1 .
$$

Likewise, using (31) we see that

$$
n_{2}=\frac{\sqrt{(1-x)(2-t(1-x))}}{G}-1 .
$$

Using (34) and (35), we have

$$
\frac{n_{1}}{n_{2}}=\frac{\sqrt{x(2-t x)}-G}{\sqrt{(1-x)(2-t(1-x))}-G} .
$$

Letting $\frac{n_{1}}{n_{2}} \equiv y$, we may write the system of two equations and two unknowns $(x$ and $y)$ formed by (33) and (36) as follows:

$$
\left\{\begin{array}{l}
y=\frac{\sqrt{x(2-t x)(1-t(1-x))}}{\sqrt{(1-x)(2-t(1-x))(1-t x)}} \\
y=\frac{\sqrt{x(2-t x)}-G}{\sqrt{(1-x)(2-t(1-x))}-G}
\end{array} .\right.
$$

The pairs of $y$ and $x$ that solve this system (given values of the parameters) are also solutions to the original system of equations $\sqrt{29}$ It is easy to see that $x=\frac{1}{2}$ and $y=1$ is a solution regardless of the value of $G$. When $x \neq \frac{1}{2}$, the value of $G$ becomes relevant. A simple change of variables, however, confirms that the system is symmetric around $x=\frac{1}{2}$. Therefore, we

${ }^{29}$ Because $y$ is the ratio $\frac{n_{1}}{n_{2}}$, to recover the exact values of $n_{1}$ and/or $n_{2}$ we use 34 and 35 . 
need only study the solutions for the case $0 \leq x \leq \frac{1}{2}$ : if $\hat{x}<\frac{1}{2}$ is part of a solution, then $1-\hat{x}>\frac{1}{2}$ is part of another solution.

We now equate both expressions and solve for $G$ to obtain:

$$
G=\frac{\sqrt{(2-t(1-x))(1-x)} \sqrt{x(2-t x)}(\sqrt{1-t x}-\sqrt{1-t(1-x)})}{\sqrt{(2-t(1-x))(1-x)} \sqrt{1-t x}-\sqrt{1-t(1-x)} \sqrt{x(2-t x)}} .
$$

Define

$$
Y(x)=\frac{\sqrt{(2-t(1-x))(1-x)} \sqrt{x(2-t x)}(\sqrt{1-t x}-\sqrt{1-t(1-x)})}{\sqrt{(2-t(1-x))(1-x)} \sqrt{1-t x}-\sqrt{1-t(1-x)} \sqrt{x(2-t x)}},
$$

so that it holds that $Y(x)=G$. It is easy to show that (given $t) Y(x)$ is strictly increasing in $x$ (over the range $0 \leq x \leq \frac{1}{2}$ ). Therefore, given $t$, the maximum $G$ such that the system 37 has a solution (other than for $x=\frac{1}{2}$ in which case $G$ is irrelevant) is

$$
\lim _{x \rightarrow \frac{1}{2}} Y(x)=\frac{(4-t)^{\frac{3}{2}} t}{2\left(8-4 t+t^{2}\right)}
$$

Therefore, the system has at least one more solution if and only if

$$
G \leq \frac{(4-t)^{\frac{3}{2}} t}{2\left(8-4 t+t^{2}\right)}
$$

We now show that when $G \leq \frac{(4-t)^{\frac{3}{2}} t}{2\left(8-4 t+t^{2}\right)}$ the system has only one solution with $x<\frac{1}{2}$. We reason by contradiction. Suppose that there were more than one solution. Consider two such solutions $\left(x_{1}, y_{1}\right)$ and $\left(x_{2}, y_{2}\right)$ with $x_{1}, x_{2}<\frac{1}{2}$. Obviously, a necessary condition for $\left(x_{1}, y_{1}\right)$ and $\left(x_{2}, y_{2}\right)$ to be different is that $x_{1} \neq x_{2}$. Assume, without loss of generality, that $x_{1}<x_{2}<\frac{1}{2}$. At these values of $x$ we must have that $Y\left(x_{1}\right)=G$ and $Y\left(x_{2}\right)=G$ but this is impossible because $Y(\cdot)$ is strictly increasing in $x$ (over the range $0 \leq x \leq \frac{1}{2}$ ). We conclude that 37 has either one solution only $\left(x=\frac{1}{2}, y=1\right.$; this happens when $\left.G>\frac{(4-t)^{\frac{3}{2}} t}{2\left(8-4 t+t^{2}\right)}\right)$ or it has three solutions (one with $x=\frac{1}{2}$, another one with $x<\frac{1}{2}$, and the symmetric 
solution with $x>\frac{1}{2}$; this happens when $\left.G \leq \frac{(4-t)^{\frac{3}{2}} t}{2\left(8-4 t+t^{2}\right)}\right)$.

Finally, we show that $x=\frac{1}{2}$ satisfies the monotonicity criterion if and only if the system has three solutions. We use the following expressions:

- Optimality condition for $p^{D}$ (see proof of Proposition 3 )

$$
\frac{(a-b c) \sqrt{4-t}}{8}\left(\frac{4\left(f+c^{D}\right)+t\left(p^{D}-c^{D}\right)}{\sqrt{2 b\left(p^{D}+f\right)^{3}}}\right)=1 .
$$

- Monotonicity condition for $x=\frac{1}{2}$ (see proof of Proposition 3 ):

$$
p^{D} \geq c^{D}+\frac{8(2-t)^{2}\left(f+c^{D}\right)}{t\left(8-t^{2}\right)} .
$$

- Condition for the system to have three solutions (as derived above):

$$
\frac{(4-t)^{3} t^{2}}{4\left(8-4 t+t^{2}\right)^{2}} \geq \frac{2 b\left(p^{D}+f\right)}{(a-b c)^{2}}
$$

The optimality condition for $p^{D}$ can be written as

$$
\frac{(4-t)}{64} \frac{\left(4\left(f+c^{D}\right)+t\left(p^{D}-c^{D}\right)\right)^{2}}{\left(p^{D}+f\right)^{2}}=\frac{\left(p^{D}+f\right) 2 b}{(a-b c)^{2}} .
$$

Therefore, we can rewrite the condition for the system to have three solutions as:

$$
\begin{aligned}
\frac{(4-t)^{3} t^{2}}{4\left(8-4 t+t^{2}\right)^{2}} & \geq \frac{(4-t)}{64} \frac{\left(4\left(f+c^{D}\right)+t\left(p^{D}-c^{D}\right)\right)^{2}}{\left(p^{D}+f\right)^{2}} \\
\frac{(4-t)^{2} t^{2}}{\left(8-4 t+t^{2}\right)^{2}} & \geq \frac{\left(4\left(f+c^{D}\right)+t\left(p^{D}-c^{D}\right)\right)^{2}}{16\left(p^{D}+f\right)^{2}} \\
\frac{4 t(4-t)}{\left(8-4 t+t^{2}\right)} & \geq \frac{\left(4\left(f+c^{D}\right)+t\left(p^{D}-c^{D}\right)\right)}{\left(p^{D}+f\right)}
\end{aligned}
$$

We would like to see that

$$
\frac{4 t(4-t)}{\left(8-4 t+t^{2}\right)} \geq \frac{\left(4\left(f+c^{D}\right)+t\left(p^{D}-c^{D}\right)\right)}{\left(p^{D}+f\right)}
$$


is satisfied when

$$
p^{D} \geq c^{D}+\frac{8(2-t)^{2}\left(f+c^{D}\right)}{t\left(8-t^{2}\right)} .
$$

Given $p^{D}, c^{D}$, and $f$, let $t^{*}$ be the value of $t$ such that both (38) and (39) are satisfied with equality. (It is easy to see that this is the same $t$.)

We now show that as $t$ increases from $t^{*}$, both (38) and (39) continue to be satisfied. To see this, consider first (38). The derivative of the lhs with respect to $t$ is

$$
\frac{d}{d t}\left(\frac{4 t(4-t)}{\left(8-4 t+t^{2}\right)}\right)=\frac{64(2-t)}{\left(8-4 t+t^{2}\right)^{2}}>0 .
$$

The derivative of the rhs with respect to $t$ is

$$
\frac{d}{d t}\left(\frac{\left(4\left(f+c^{D}\right)+t\left(p^{D}-c^{D}\right)\right)}{\left(p^{D}+f\right)}\right)=\frac{p^{D}-c^{D}}{p^{D}+f}>0 .
$$

Because at $t^{*}(38)$ is satisfied with equality, we have that

$$
\frac{4 t^{*}\left(4-t^{*}\right)}{\left(8-4 t^{*}+t^{* 2}\right)}=\frac{\left(4\left(f+c^{D}\right)+t^{*}\left(p^{D *}-c^{D}\right)\right)}{\left(p^{D *}+f\right)}
$$

or

$$
\left(8-4 t^{*}+t^{* 2}\right)=\frac{4 t^{*}\left(4-t^{*}\right)\left(p^{D}+f\right)}{4\left(f+c^{D}\right)+t^{*}\left(p^{D}-c^{D}\right)} .
$$

We use this expression to derive the following:

$$
\begin{aligned}
\left.\frac{d}{d t}\left(\frac{4 t(4-t)}{\left(8-4 t+t^{2}\right)}\right)\right|_{t^{*}} & =\frac{64\left(2-t^{*}\right)}{\left(8-4 t^{*}+t^{* 2}\right)^{2}}=\frac{64\left(2-t^{*}\right)}{8-4 t^{*}+t^{* 2}} \frac{4\left(f+c^{D}\right)+t^{*}\left(p^{D}-c^{D}\right)}{4 t^{*}\left(4-t^{*}\right)\left(p^{D}+f\right)} \\
& >\frac{64\left(2-t^{*}\right)}{8-4 t^{*}+t^{* 2}} \frac{\left(p^{D}-c^{D}\right)}{4\left(4-t^{*}\right)\left(p^{D}+f\right)}=\frac{64\left(2-t^{*}\right)}{4\left(4-t^{*}\right)\left(8-4 t^{*}+t^{* 2}\right)} \frac{p^{D}-c^{D}}{p^{D *}+f} \\
& =\left.\frac{64\left(2-t^{*}\right)}{4\left(4-t^{*}\right)\left(8-4 t^{*}+t^{* 2}\right)} \frac{d}{d t}\left(\frac{\left(4\left(f+c^{D}\right)+t\left(p^{D}-c^{D}\right)\right)}{\left(p^{D}+f\right)}\right)\right|_{t^{*}} \\
& >\left.\frac{d}{d t}\left(\frac{\left(4\left(f+c^{D}\right)+t\left(p^{D}-c^{D}\right)\right)}{\left(p^{D}+f\right)}\right)\right|_{t^{*}}
\end{aligned}
$$

The last inequality follows from

$$
\frac{64\left(2-t^{*}\right)}{4\left(4-t^{*}\right)\left(8-4 t^{*}+t^{* 2}\right)} \geq 1
$$


Therefore at $t^{*}$ :

$$
\frac{d}{d t}(l h s)>\frac{d}{d t}(r h s) .
$$

Finally, note that the second derivative of the lhs wrt to $t$ is

$$
\frac{d^{2}}{d t^{2}}(\text { lhs })=\frac{64(8-3(4-t) t)}{\left(8-4 t+t^{2}\right)^{3}}
$$

which is positive for $0 \leq t \leq \frac{2}{3}$, and note as well that $\frac{d^{2}}{d t^{2}}(r h s)=0$. Therefore, as $t$ grows from $t^{*},(38)$ is satisfied.

Let's now consider (39). The derivative of the rhs with respect to $t$ is

$$
\frac{d}{d t}\left(\frac{8(2-t)^{2}\left(f+c^{D}\right)}{t\left(8-t^{2}\right)}\right)=\frac{8\left(c^{D}+f\right)\left(-32+t^{2}(20-(8-t) t)\right)}{t^{2}\left(8-t^{2}\right)^{2}},
$$

which is less than zero for $0 \leq t \leq \frac{2}{3}$. Therefore, as $t$ grows from $t^{*}, 39$ becomes less and less stringent.

Suppose now that the parameter values are such that the monotonicity condition for $x=\frac{1}{2}$ hold. We must have

$$
p^{D} \geq c^{D}+\frac{8(2-t)^{2}\left(f+c^{D}\right)}{t\left(8-t^{2}\right)} .
$$

Now, reduce $t$ so that this condition holds with equality $(t$ must be reduced because the rhs is decreasing in $t$; moreover, equality can always be attained because the limit of the rhs as $t \downarrow 0$ is $\infty$ ). At this point, the condition for the system to have three solutions is also satisfied with equality. The above argument shows that at the original, higher, $t$ we must have that

$$
\frac{4 t(4-t)}{\left(8-4 t+t^{2}\right)} \geq \frac{\left(4\left(f+c^{D}\right)+t\left(p^{D}-c^{D}\right)\right)}{\left(p^{D *}+f\right)}
$$

and thus 38 is satisfied. We conclude that whenever the monotonicity condition for $x=\frac{1}{2}$ holds, the system has three solutions. The same argument can be made to show that when the system has three solutions, then the monotonicity condition for $x=\frac{1}{2}$ holds.

Proof of Proposition 4. The left hand side of the expression in (26) for $p^{D}=p^{D e}$ is equal to $1+\frac{t}{4}\left(\sqrt[3]{\frac{(4-t)(a-b c)^{2}}{8 b\left(f+c^{D}\right)}}-1\right)$, which exceeds 1 because it has been assumed that 
$\frac{(a-b c)^{2}}{2 b} \geq Z^{\prime}$ and we have shown in the proof of Proposition 1 that $Z^{\prime}>\frac{4\left(f+c^{D}\right)}{4-t}$, so that inequality $\frac{(a-b c)^{2}}{2 b}>\frac{4\left(f+c^{D}\right)}{4-t}$ is fulfilled. Therefore, it follows that $p^{D e}<p^{D *}$, and hence we have that $n^{e}>n^{* *}$. In addition, it is easy to see that $p^{D e}>c^{D}$ because it holds that $\frac{(a-b c)^{2}}{2 b}>\frac{4\left(f+c^{D}\right)}{(4-t)}$.

Proof of Remarks 2 and 3. The expressions for $W^{\text {com }}\left(N^{*}\right)$ and $W^{\text {inc }}\left(n^{* *}\right)$ are derived exactly as in Sections 3.1 and 4.1 , respectively.

We obtain $\tilde{W}^{i n c}$ as follows. Under asymmetric incompatibility only platform 1 has users and developers in equilibrium. Every developer who traded with platform 1 makes profits

$$
\tilde{\pi}_{1}^{i n c}=\frac{x_{1}\left(2-t x_{1}\right)}{2 b}\left(\frac{a-b c}{n_{1}+1}\right)^{2} .
$$

Because the market is covered $\left(x_{1}=1\right)$, profits are:

$$
\tilde{\pi}_{1}^{i n c}=\frac{(2-t)}{2 b}\left(\frac{a-b c}{n_{1}+1}\right)^{2}=(2-t) A \frac{1}{\left(n_{1}+1\right)^{2}},
$$

where $A=\frac{(a-b c)^{2}}{2 b}$.

Welfare under asymmetric incompatibility is:

$$
\begin{aligned}
\tilde{W}^{i n c} & =\underbrace{\int_{0}^{1}(1-t s) A\left(\frac{\widetilde{n}_{1}^{*}}{\widetilde{n}_{1}^{*}+1}\right)^{2} d s}_{\text {Users' surplus }}+\underbrace{\left((2-t) A \frac{1}{\left(\widetilde{n}_{1}^{*}+1\right)^{2}}-f-c^{D}\right)}_{\text {Platform's profits }} \widetilde{n}_{1}^{*} \\
& =\left(1-\frac{t}{2}\right) A\left(1-\frac{1}{\left(\widetilde{n}_{1}^{*}+1\right)^{2}}\right)-\left(f+c^{D}\right) \widetilde{n}_{1}^{*} .
\end{aligned}
$$

The equilibrium number of developers $\widetilde{n}_{1}^{*}$ solves

$$
\tilde{\pi}_{1}^{i n c}-f-\widetilde{p}_{1}^{D}=0
$$

or

$$
(2-t) A \frac{1}{\left(\widetilde{n}_{1}^{*}+1\right)^{2}}-f-\widetilde{p}_{1}^{D}=0,
$$

where $\widetilde{p}_{1}^{D}$ is the solution to the constrained optimization programme presented in Section 
5.2. Therefore,

$$
\widetilde{n}_{1}^{*}=\sqrt{\frac{A(2-t)}{f+\widetilde{p}_{1}^{D}}}-1 .
$$




\section{References}

[1] Armstrong, M. (2006), "Competition in Two-Sided Markets," RAND Journal of Economics $37(3), 668-691$.

[2] Caillaud, B. and Bruno Jullien (2001), "Competing Cybermediaries," European Economic Review 45(4-6), 797-808.

[3] Caillaud, B. and Bruno Jullien (2003), "Chicken and Egg: Competition among Intermediation Service Providers," RAND Journal of Economics 34(2), 521-552.

[4] Carrillo, J. and Guofu Tan (2006), "Platform Competition: The Role of Multi-homing and Complementors," NET Institute Working Paper No 06-30.

[5] Church, J. and N. Gandal (1993), "Complementary Network Externalities and Technological Adoption," International Journal of Industrial Organization, No. 11, pp. 239-260.

[6] Crémer, J., Patrick Rey and Jean Tirole (2000), "Connectivity in the Commercial Internet," Journal of Industrial Economics, 48, pp. 433-472.

[7] Economides, N. (1989), "Desirability of Compatibility in the Absence of Network Externalities," The American Economic Review, Vol. 79, No. 5, pp. 1165-1181.

[8] Economides, N. and Fredrick Flyer (1997), "Compatibility and Market Structure for Network Goods," Mimeo, New York University.

[9] Evans, D. S. (2003), "The Antitrust Economics of Multi-Sided Platform Markets," Yale Journal on Regulation 20(2), 325-382.

[10] Evans, D. S. and Richard Schmalensee (2007), "The Industrial Organization of Markets with Two-Sided Platforms," Issues on Competition Law and Policy, W.D. Collins ed.

[11] Hagiu, A. (2006a), "Pricing and Commitment by Two-Sided Platforms," RAND Journal of Economics 37(3), 720-737.

[12] Hagiu, A. (2006b), "Proprietary vs. Open Two Sided Platforms and Social Efficiency," AEIBrookings Joint Center Working Paper 06-12.

[13] Hagiu, A. (2009), "Two-Sided Platforms: Product Variety and Pricing Structures," Journal of Economics \& Management Strategy, 18(4).

[14] Katz, M. L. and Carl Shapiro (1985), "Network Externalities, Competition, and Compatibility," American Economic Review 75(3), 424-440.

[15] Katz, M. L. and Carl Shapiro (1986), "Product Compatibility Choice in a Market with Technological Progress," Oxford Economic Papers, New Series, Vol. 38, Supplement: Strategic Behaviour and Industrial Competition, pp. 146-165.

[16] Katz, M. L. and Carl Shapiro (1994), "Systems Competition and Network Effects," The Journal of Economic Perspectives, Vol. 8, No. 2, pp. 93-115. 
[17] Kreps, D. M. and Jose A. Scheinkman (1983), "Quantity Precommitment and Bertrand Competition Yield Cournot Outcomes," Bell Journal of Economics 14(2), 326-337.

[18] Malueg, D. A. and Marius Schwartz (2006), "Compatibility Incentives of a Large Network Facing Multiple Rivals," Journal of Industrial Economics, 54, pp. 527-567.

[19] Miao, Chun-Hui (2007), "Compatibility in Two-Sided Markets," Mimeo, University of South Carolina.

[20] Matutes, C. and Pierre Regibeau (1988), "“Mix and Match:" Product Compatibility without Network Externalities," RAND Journal of Economics 9(2), 221-234.

[21] Novshek, William (1980), "Equilibrium in Simple Spatial (Differentiated Product) Models," Journal of Economic Theory 22, 313-326.

[22] Orman, Wafa Hakim (2008), "The Effects of Compatibility on Competition between Proprietary and Open Two-Sided Platforms." Working Paper (3/28/2008).

[23] Rochet, J-C. and Jean Tirole (2002), "Cooperation among Competitors: Some Economics of Payment Card Associations," RAND Journal of Economics 33(4), 1-22.

[24] Rochet, J-C. and Jean Tirole (2003), "Platform Competition in Two-Sided Markets," Journal of the European Economic Association 1(4), 990-1029.

[25] Rochet, J-C. and Jean Tirole (2006), "Two-Sided Markets: A Progress Report," RAND Journal of Economics 37(3), 645-667.

[26] Spulber, Daniel F. (1996), "Market Making by Price Setting Firms." Review of Economic Studies 63, 559-580.

[27] Spulber, Daniel F. (1999), Market Microstructure: Intermediaries and the Theory of the Firm. Cambridge University Press, New York.

[28] Spulber, Daniel F. (2006), "Firms and Networks in Two-Sided Markets." In Terrence Hendershott, Ed., Handbooks in Information Systems, Vol. 1. 137-200.

[29] Suzumura K. and K. Kiyono (1987), "Entry Barriers and Economic Welfare," Review of Economic Studies 54(1), 157-167.

[30] Weyl, Glen (2009), "A Price Theory of Multi-Sided Platforms," American Economic Review. Forthcoming. 